\title{
An Integrated Method for Detecting Micro RNA Target Proteins through Reverse-phase Protein Arrays
}

Jiawen Zhu' ${ }^{1}$, Song $\mathbf{W u}^{1}$ and Jie Yang ${ }^{2 *}$

${ }^{1}$ Department of Applied Mathematics and Statistics, Stony Brook University, Stony Brook, NY 11790, USA

${ }^{2}$ Department of Preventive Medicine, Stony Brook University, Stony Brook, NY 11790, USA

\begin{abstract}
Objective: Understanding functions of microRNAs (or miRNAs), particularly their effects on protein degradation, is biologically important. Emerging technologies, including the reverse- phase protein array (RPPA) for quantifying protein concentration and RNA-seq for quantifying miRNA expression, provide a unique opportunity to study miRNA-protein regulatory mechanisms. One naïve way to analyze such data is to directly examine the correlation between the raw miRNA measurements and protein concentrations estimated from RPPA. However, the uncertainty associated with protein concentration estimates is ignored, which may lead to less accurate results and significant power loss.
\end{abstract}

Methods: We propose an integrated nonlinear hierarchical model for detecting miRNA targets through original RPPA intensity data. This model is fitted within a maximum likelihood framework and the correlation test between miRNA and protein is assessed using Wald tests. We compare this model and the simple method through extensive simulation studies and a real dataset from the Cancer Genome Atlas (TCGA) project.

Results: This integrated method is shown to have consistently higher power than the simple method, especially when sample sizes are limited and when the RPPA intensity levels are close to the boundaries of imaging limits.

Conclusions: Our proposed method is powerful in detecting miRNA's protein target through RPPA. We recommend this method in practice.

Keywords: MicroRNA; Reverse-phase Protein Arrays; Nonlinear mixed model; Micro RNA target

\section{Introduction}

MicroRNA (miRNA) is a set of small, non-coding RNA molecules that can post-transcriptionally regulate a broad range of gene expression in both plants and animals. They have been suggested to be involved in many important biological processes, such as normal physiological development and disease onsets. In the past decade, many efforts have been put to search for the miRNA targets [1]. Although our understanding on some miRNAs has been dramatically improved, as of today, the targets of many others remain largely unknown. Therefore, powerful methods for efficient detection of miRNA targets are still in great need.

In general, miRNA regulates the expression of its target genes through two mechanisms-mRNA degradation or translation inhibition. That is, if a miRNA and its target gene can complement extensively, the miRNA-mRNA target may form a double-strand RNA (dsRNA) structure, after which, the mRNA can be cleaved and degraded to lower the mRNA expression and subsequently protein expression $[2,3]$. On the other hand, if a miRNA and its target can only complement partially, the target mRNA will not be directly degraded but its translation may be repressed $[4,5]$. So, in both mechanisms, the total protein level relating to the miRNA targets would be reduced, resulting in their functional losses.

Based on the phenomenon that the sequences of miRNAs and their target genes complement to each other, or at least partially, one way of the miRNA target identification is through in silico prediction. Several software tools have been developed for such purpose, each with its own unique feature. For example, miRanda scored the likelihoods of mRNA down-regulation according to a regression model that is trained on sequence and contextual features of the predicted miRNA::mRNA duplex [6], and Target Scan studies on the miRNA::mRNA duplex interactions according to a thermodynamics-based modeling and comparative sequence analysis [7]. Based on these computational tools, several databases with predicted miRNA targets have been generated (microRNA.org and targetscan.org). However, one major limitation is that they all suffer from large percentage of false positives, which hinder their practical usage.

Another popular way to determine the miRNA targets is through experimental data by measuring downstream effects of miRNAs. Since miRNAs can induce protein reduction via both functional mechanisms, protein expression seems to be the right mark. However, due to difficulties in high- throughput quantification of protein expression but relative ease in that of mRNA, conventionally, scanning of the miRNA targets is mainly through testing negative correlations between miRNAs and mRNAs. For example, high-throughput techniques, such as miRNA and mRNA gene microarray, can be applied to measure their expression levels, and then the correlations analyses can be conducted subsequently to filter out miRNA-mRNA pairs that show significant negative correlations as potential candidates for further analyses [8]. More recently, with the advent and rapid advance of sequencing techniques, the miRNA sequencing (miRNA-seq) and RNA sequencing (RNA-seq) platforms have become more and more

*Corresponding author: Jie Yang, Department of Preventive Medicine, Stony Brook University, Stony Brook, NY 11790, USA, Tel: 631-444-2191; Fax: +1 631444-7525; E-mail: jie.yang@stonybrookmedicine.edu

Received November 03, 2014; Accepted November 18, 2014; Published January 01,2015

Citation: Zhu J, Wu S, Yang J (2015) An Integrated Method for Detecting Micro RNA Target Proteins through Reverse-phase Protein Arrays. J Comput Sci Syst Biol 8: 012-033. doi:10.4172/jcsb.1000166

Copyright: @ 2015 Zhu J, et al. This is an open-access article distributed under the terms of the Creative Commons Attribution License, which permits unrestricted use, distribution, and reproduction in any medium, provided the original author and source are credited. 
popular for the quantification of the miRNA and RNA expressions. The main drawback for the miRNA/mRNA correlation analyses is that they can only identify miRNA targets that may change at mRNA levels, but is determined to fail for those modulated through translation inhibition. Therefore, miRNA/mRNA correlation analysis is only able to find partial targets of miRNAs.

It is only until recently that a new technology, called the reverse phase protein array (RPPA) or protein lysate array has been developed to simultaneously quantify the protein expression in a large sample cohort [9]. In contrast to the usual array design that quantifies expression levels of multiple genes in one sample, the RPPA measures the protein expression levels of many samples on one array. Usually, a 2 -fold serial dilution of samples is used to avoid signal saturation for very high protein concentration. Since the RPPA data are measured through image signals, which are characterized by background noises at the low end and saturation signals at the high end, a sigmoid-like response curve model has been used to estimate protein concentration [10,11]. Additionally, some flexible nonparametric joint sample models have also been proposed [12]. In principle, we can directly use the protein concentrations estimated from these methods to correlate with miRNA expression for miRNA target identification. However, in this case, the uncertainties associated with the protein concentration estimates are ignored, leading to less accurate and less powerful inference.

In this article, we propose an integrated hierarchical model to detect miRNA targets based on protein expression data measured by the recently emerged RPPA and high throughput miRNA- seq data. Extensive Monte Carlo simulations have been performed to examine the performance of our proposed model under different scenarios. This model was further applied to a real dataset from TCGA to demonstrate its practical use.

\section{Methods}

\section{PPA}

To quantify the protein expression on a RPPA array, a sigmoidal model is commonly assumed to describe the relationship between the intensity level and the protein concentration with additive error $[10,13]$ :

$$
y_{i j}=g\left(x_{i}, l_{j}, \beta\right)=\beta_{1}+\frac{\beta_{2}}{1+2^{-\beta_{3}\left(x_{i}+l_{j}\right)}}+\varepsilon_{i j}
$$

where $y_{i j}$ is the gray-level intensity from sample $i$ at $j$ th dilution, $i=1, \ldots$, $I$ and $j=1, \ldots, J, x_{i}$ is the binary logarithm of the median effective protein concentration level $\left(\mathrm{EC}_{50}\right)$, a single quantity per dilution series to represent the concentration of the protein, $x_{i}+l j$ is the binary logarithm of the protein concentration after $j t$ dilution where $l_{j}=\frac{(1+J)}{2}-j$, $\varepsilon_{i j}$ is the error term assumed to have a normal distribution with mean 0 and variance $\sigma_{1}^{2}$, and $\boldsymbol{\beta}=\left\{\beta_{1}, \beta_{2}, \beta_{3}\right\}$. Since $\beta_{1}=\lim _{x_{i} \rightarrow-\infty} E\left(y_{i j}\right)$ and $\beta_{1}+\beta_{2}=\lim _{x_{i} \rightarrow-\infty} E\left(y_{i j}\right), \beta_{1}$ is interpreted as the lowest intensity level without noise, and $\beta_{2}$ is the increment from the lowest to the highest intensity or the saturation level.

To estimate the relative protein concentration in RPPA, one algorithm based on logistic model fitting used by $\mathrm{Hu}$ [12] is given as follows:

The initial intensity data are first transformed as

$$
y_{\text {linear }_{i j}}=\operatorname{logit}_{2} \frac{y_{i j}-\min (Y)}{\operatorname{range}(Y)}
$$

and initial $\boldsymbol{\beta}$ are set as

$\hat{\beta}_{1}^{(0)}=\min (Y), \hat{\beta}_{2}^{(0)}=\max (Y)-\min (Y), \hat{\beta}_{3}^{(0)}=\hat{\beta}_{2}^{(0)} /(J-1), Y=\left\{y_{i j} \mid i=1, \ldots, I\right.$ and $\left.j=1, \ldots, J\right\}$, $\operatorname{range}(Y)=\max (Y)-\min (Y)$.

The initial median effective protein concentration level $x_{i}$ are estimated by using:

$$
x_{i}=\text { median } \frac{\left(y_{\text {linear }_{i}}\right)}{\hat{\beta}_{3}}+l_{j}
$$

where $y_{\text {linear }}$ is the mean value among $\left\{y_{\text {linear }_{i}}\right\}$

To update the parameters $\left(\hat{\beta}_{1}^{(0)}, \hat{\beta}_{2}^{(0)}, \hat{\beta}_{3}^{(0)}\right)$ in the nonlinear model, the nonlinear least-squares estimates of $\left(\hat{\beta}_{1}^{(1)}, \hat{\beta}_{2}^{(1)}, \hat{\beta}_{3}^{(1)}\right)$ were calculated based on the following model [14]:

$$
y_{i j}=g\left(x_{i}, l_{j}, \beta\right)=\beta_{1}+\frac{\beta_{2}}{1+2^{-\beta_{3}\left(x_{i}-l_{j}\right)}}+\varepsilon_{i j}
$$

After obtaining $\hat{\beta}_{1}^{(1)}, \hat{\beta}_{2}^{(1)}, \hat{\beta}_{3}^{(1)}$, the nonlinear least-square method is used again to update the relative protein level $\boldsymbol{X}=\left\{x_{i}, i=1 \ldots I\right\}$ and $\sigma_{1}^{2}$. This iteration continues until convergence.

\section{A naïve model for correlating miRNA and protein expression}

Since $\boldsymbol{X}=\left\{x_{i}, i=1,2, \ldots I\right\}$, the $\log 2$ transformation of the median effective protein concentration levels, can be estimated from the RPPA, a straightforward way to examine the relationship between miRNA and protein expression levels is through Pearson's correlation coefficients or simple linear regression models, which is referred as the naïve model in this article.

The linear relationship between protein and miRNA in the naïve model can be expressed as: $x_{i}=f\left(Z_{i}\right)=\alpha_{1}+\alpha_{2} Z_{i}+\eta_{i}$, where $\eta_{i} \sim N\left(0, \sigma_{0}^{2}\right)$ and $\left\{z_{i}, i=1,2 \ldots I\right\}$ are log-transformed expression levels of a specific miRNA from sample $i=1,2 \ldots I$.

The parameter estimates $\hat{\alpha}_{1}, \hat{\alpha}_{2}, \hat{\alpha}_{0}$ can be calculated by using linear regression. $\hat{\alpha}_{2}$ is our parameter of interest, describing the correlation between a miRNA and protein pair. We can test $H_{0}: \alpha=0$ to determine if a particular pair of miRNA and protein is related or not.

\section{A hierarchical model for correlating miRNA and protein expression}

Although the naïve method is straightforward, uncertainty associated with protein concentration estimates is ignored. As demonstrated in later sections, it leads to less accurate results and significant power loss. Here we propose a nonlinear hierarchical model for studying the relationship between miRNA and protein expression, in which the correlation analysis is integrated with the estimation of protein concentration. The model is given as follows:

$$
\begin{aligned}
& y_{i j}=g\left(x_{i}, l_{j}, \beta\right)=\beta_{1}+\frac{\beta_{2}}{1+2^{-\beta_{3}\left(x_{i}-l_{j}\right)}}+\varepsilon_{i j} \\
& x_{i}=f\left(Z_{i}\right)+\eta_{i}, \\
& \eta_{i} \sim N\left(0, \sigma_{0}^{2}\right), \varepsilon_{i j} \sim N\left(0, \sigma_{0}^{2}\right), \eta_{i} \perp \varepsilon_{i j}
\end{aligned}
$$

Here (.) is a general function to describe how $x_{i}$, the protein level, and $z_{i}$, the miRNA expression level, is related. $\boldsymbol{\beta}=\left\{\beta_{1{ }^{\prime},}, \beta_{3}\right\}$ is the parameter vector of the response curve function $g($.$) . To directly$ 
compare with the naïve model, we assume $(z i)$ to be linear in equation (6), that is, $f\left(Z_{i}\right)=\alpha_{1}+\alpha_{2} Z_{i}$. We further assume that the two error terms, $\eta_{i}$ and $\varepsilon_{i j}$ are independent of each other. In this hierarchical framework, the relationship between miRNA and protein expression level can be estimated without explicitly quantifying the protein concentration based on intensity data first. This model is referred as the integrated model hereinafter.

The likelihood function for $\boldsymbol{Y}$ and $\boldsymbol{Z}$ can be written as a joint probability function

$$
L\left(\phi, \sigma_{0}, \sigma_{1} \mid Y, Z\right)=\prod_{\substack{i=1,2 \ldots I \\ j=1,2, \ldots J}} \int_{-\infty}^{+\infty} f_{\varepsilon_{i j}}\left(y_{i j} \mid Z_{i}, \phi, \eta_{i}, \sigma_{1}\right) q_{\eta_{i}}\left(\eta_{i} \mid \sigma_{0}\right) d \eta_{i}
$$

where $\boldsymbol{\phi}=\left\{\alpha_{1}, \alpha_{2}, \beta_{1}, \beta_{2}, \beta_{3}\right\}$ is a vector including parameters in function $f($.$) and g(),. \quad \boldsymbol{Y}=\left\{y_{i j} \mid i=1,2 \ldots I, j=1,2 \ldots J\right\}$ representing the RPPA intensity levels and $\boldsymbol{Z}=\left\{Z_{i} \mid i=1,2 \ldots I\right\}$ representing the log-transformed miRNA expression levels.

\section{Computational algorithm}

The unknown parameters $\phi=\left\{\alpha_{1}, \alpha_{2}, \beta_{1}, \beta_{2}, \beta_{3}\right\}$ can be estimated within the maximum likelihood framework. The adaptive Gaussian quadrature method is used to approximate the integral and the dual quasi-Newton method can be further applied in maximizing the likelihood function [15-17]. Our computational algorithm is illustrated in Figure 1. This algorithm is implemented in SAS nlmixed procedure and a SAS macro is available upon request to fit the integrated model.

Based on the idea of Hermite integration for function $(x)$

$$
\int_{-\infty}^{+\infty} f(x) d x=\sum_{k=1}^{m} w_{k} e^{x_{k}^{2}} f\left(x_{k}\right)
$$

Our likelihood function can be written as

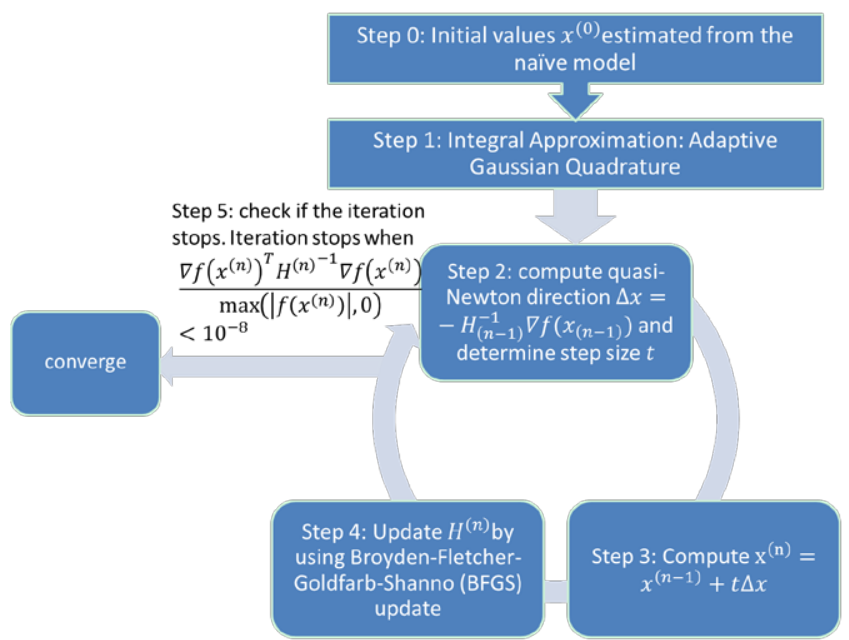

Figure 1: Flow chart of computational algorithm to fit the integrated mode based on adaptive Gaussian quadrature method and dual quasi-Newton algorithm. Step 0: estimate the initial value of $\phi,_{0}, \sigma_{1}$, denoted as $x(0)$, by using the naïve model; Step 1: generate the approximate likelihood function by using adaptive Gaussian Quadrature method; Step 2: compute the quasi-Newton direction $\Delta x$, determine the step size $t$ to satisfy the Goldstein conditions: Step 3: update parameters $x$ value; Step 4: update Hessian matrix; Step 5: check if the iteration stops. If not, go to Step 2, if yes, the iteration stops. A grid searching with center from the estimates of naïve model was applied in our algorithm.

$$
\begin{aligned}
& L\left(\phi, \sigma_{0}, \sigma_{1} \mid Y, Z\right)=\prod_{\substack{i=1,2, \ldots I \\
j=1,2, \ldots J}} \int_{-\infty}^{+\infty} f_{\varepsilon_{i j}}\left(y_{i j} \mid Z_{i}, \phi, \eta_{i}, \sigma_{1}\right) q_{\eta_{i}}\left(\eta_{i} \mid \sigma_{0}\right) d \eta_{i} \\
& \approx \prod_{\substack{i=1,2, I \\
j=1,2, \ldots J}} \sqrt{2}\left|\Gamma_{i}\right|^{\frac{1}{2}} \sum_{k=1}^{m} f_{\varepsilon_{j}}\left(\left.y_{i j}\left|Z_{i}, \phi, \sqrt{2}\right| \Gamma_{i}\right|^{\frac{1}{2}} x_{k}+\hat{\eta}_{i}, \sigma_{1}\right) q_{\eta_{i}}\left(\sqrt{2}\left|\Gamma_{i}\right|^{\frac{1}{2}} x_{k}+\hat{\eta}_{i} \mid \sigma_{0}\right) w_{k} e^{x_{k}^{2}}
\end{aligned}
$$

$m$ is the number of quadrature points which is set to 5 in our analysis. $x_{k}$ and $w_{k}$ denote the standard Gauss-Hermite abscissas and weights; $\hat{\eta} i$ minimizes

$$
-\log \left(f_{\varepsilon_{i j}}\left(y_{i j} \mid Z_{i}, \phi, \eta_{i}, \sigma_{1}\right) q_{\eta_{i}}\left(\eta_{i} \mid \sigma_{0}\right)\right)
$$

and $\Gamma_{i}$ is the Hessian matrix from the minimization.

With the approximate likelihood function, we employed the quasiNewton algorithm for parameter estimation. Unlike the Newton algorithm, approximate Hessian matrix was used instead of true Hessian matrix in the quasi-Newton algorithm. The step size $t$ was calculated by quadratic interpolation and cubic extrapolation and BFGS method was used to update the approximate Hessian matrix. A grid searching with center from the estimates of Naïve model was applied in our algorithm.

\section{Hypothesis testing}

Since it is expected that miRNA negatively regulate the protein level of its target gene, to test if there is a significant relationship between a specific pair of miRNA and protein, the hypothesis test can set up as a one-sided test:

$$
H_{0}: \alpha_{2}=0 \text { vs } H_{a}: \alpha_{2}<0
$$

Once the maximum likelihood estimates are obtained, a likelihood ratio test (LRT), a Wald test or a Score test can be constructed. However, LRT can be very time consuming and is not appropriate for one-sided test. For Score test, the confidence interval of $\alpha_{2}$ is difficult to be calculated. Thus Wald tests were used in our simulation and real data example, and its test statistic is:

$$
T . S .=\frac{\hat{\alpha}_{2}}{I(\hat{\phi})_{\alpha_{2}}^{\frac{1}{2}}} \sim \mathrm{N}(0,1) \text { under } H_{0} \text {, where }(\boldsymbol{\phi}) \text { represent the fisher }
$$

information matrix of the likelihood function. The null hypothesis was rejected when p-value was above 0.05 in our simulation study.

\section{Simulation studies}

Extensive simulation studies were carried out to examine the performance of our proposed integrated model and to compare with the naïve model approach. Protein intensities were generated by using a sigmoidal response curve (Figure 2a). And a typical miRNA expression distribution in the TCGA data was borrowed in this simulation to mimic the real data and generate protein $\mathrm{EC}_{50 \mathrm{~s}}$ (Figure $2 \mathrm{~b}$ ). Also, the true values of $\left\{\beta_{{ }_{1} 2}, \beta_{3}, \sigma_{0}, \sigma_{1}\right\}$ were set as $\{50,30000,1,1$, and 300$\}$ to mimic parameter values estimated from a real TCGA ovarian cancer data set. Different strengths of correlation between miRNA and protein expression levels, as characterized by $\alpha_{2}$, were examined in a range from 0 , which represents the null hypothesis, to -1.5 , which yields the power of 1 for the integrated method. In order to investigate the performance of two models with protein intensity values located in different areas of the sigmoidal curve, $\alpha_{1}$ was set as 0 and 5 corresponding to the middle part and upper part of sigmoidal curve, respectively. The upper part of a sigmoidal curve corresponds to a scenario where most of intensity levels are close to the saturation point. The RPPA intensity levels range between 10 and 30100. An illustration of the sigmoidal curve used to generate simulated data was showed in Figure 2a. The locations of 

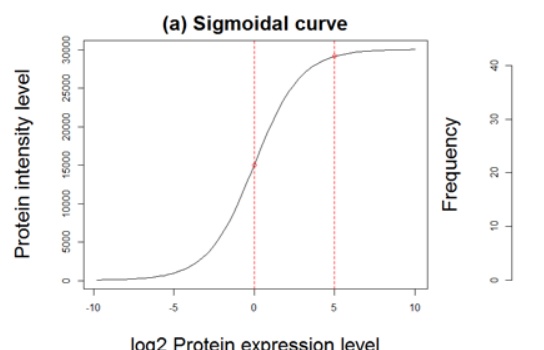

Figure 2: An illustration of (a) a sigmoidal shape response curve. When $\alpha_{1}$ was set to be 0 , the center of the EC50s would located at 0 ; When $\alpha_{1}$ was set to be 5 , the center of EC50s would located at 5; (b) distribution of a typical miRNA expression in the TCGA data we used in the simulation.
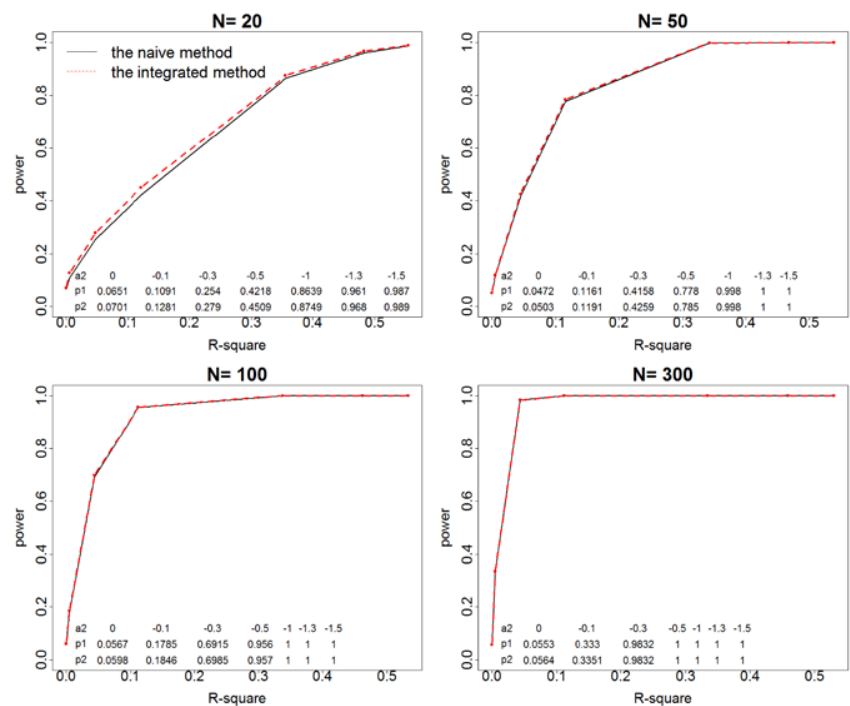

Figure 3: Power curve of the naïve model (solid line) and the integrated mode (dashed line) according to different simulation scenarios: sample size ranged from 20 to 300 and the protein intensities were located in the middle part of a sigmoidal curve. Detection powers (type I error if $\alpha_{2}=0$ ) denoted by $p_{1}$ and $p_{2}$ under different correlation strengths were report on the bottom of each plot for the naïve and integrated models, respectively. Both models can well control the pre-specified type-I error when sample size were bigger than 50 . Two models had similar detection performance, especially when sample size increased.

protein intensity center were marked by circles. If simulated intensity values are beyond the imaging boundary, they would be replaced with the boundary value with small error (Gaussian distributed with mean 0 and standard deviation 5). 1000 simulations were carried out for each parameter setting under different sample sizes $(\mathrm{N}=20,50,100$ and 300$)$. Generally, there are 5 diluted samples in one dilution series, so $J=5$ were used in our simulation setting. Pre-specified Type I error was set to be 0.05 .

The false positive rates and detection powers for miRNA targets for both the integrated model and the naive model under different sample sizes were shown in Figures 3 and 4 . It is clear that when there was no relationship between miRNA and protein $(\alpha=0)$, both models can well control the pre-specified type-I error when sample size were bigger than 50 . The integrated model was consistently more powerful than the naive model, especially when the RPPA intensity levels are
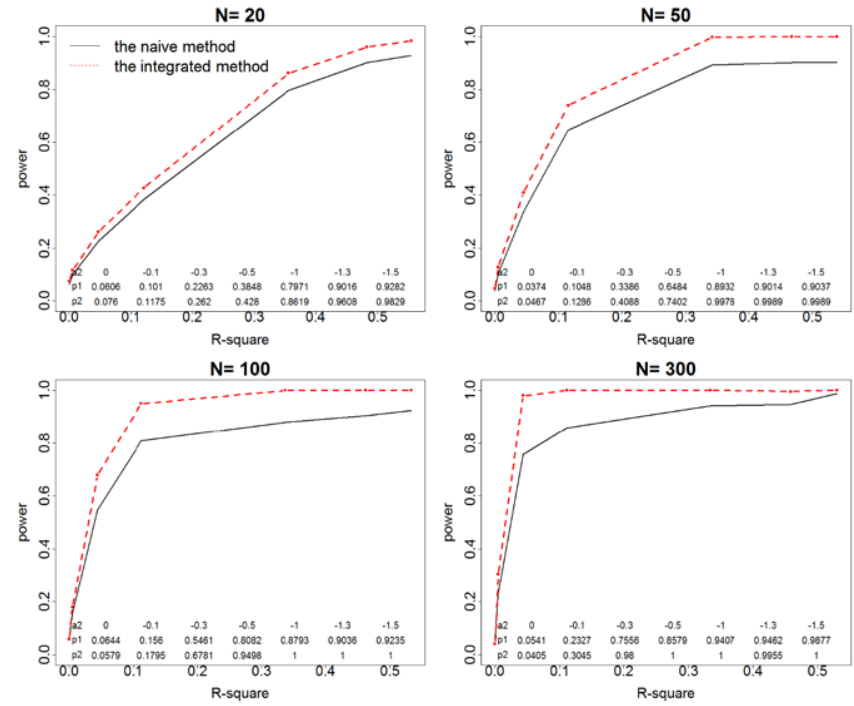

Figure 4: Power curves of the naïve model (solid line) and the integrated model (dashed line) according different simulation scenarios: sample size ranged from 20 to 300 and the protein intensities were located in the upper part of a sigmoidal curve. Detection powers (type I error if $\alpha_{2}=0$ ) denoted by $p_{1}$ and $p_{2}$ under different correlation strength were report on the bottom of each figure for the naïve and integrated models, respectively. Both models can well control the pre-specified type-l error when sample size were bigger than 50 . The integrated model was consistently more powerful than the naïve model.

close to the boundaries of imaging limits (Figure 4). Table A1-A2 in appendix listed the detailed point estimates of all parameters and their corresponding standard errors. Figure A1 and A2 in appendix illustrated the variations of the point estimates of $\alpha_{2}$ under different simulation settings. The integrated model consistently yielded parameter estimates of $\alpha_{2}$ with similar or much less standard errors than those from the naive model. When the RPPA intensities reached the upper flatter part of the sigmoidal curve, which caused information loss because of intensity level truncation at the saturation points, both the naive and the integrated method over-estimated $\beta$, which represents the lower imaging limits. However, in this situation the integrated method still had a much larger detection power than the naïve method (Figure 4).

\section{Analysis of TCGA Ovarian Cancer Data}

Both models were applied onto an ovarian cancer dataset from the TCGA project. In this dataset, there were 333 ovarian cancer samples with both miRNA and RPPA data available. 352 miRNAs having more than $50 \%$ of non-zero counts and 165 proteins were included in our analyses.

The results from both naïve and integrated models on predicting miRNA targets were reported in Table 1. False Discover Rate (FDR) at $10 \%$ was used to adjust for multiple testing [18]. The integrated model approach we proposed found 1106 potential miRNA-protein pairs, 797 of which were on non-phosphorylated protein array. 822 pairs were found on non-phosphorylated protein array: 250 out of them were found by integrated model only and 25 pairs were found by naïve model only. Integrated model found significantly more number of potential miRNA-protein pairs $(\mathrm{P}<0.0001$ according to McNemar's test). Furthermore, we compared our results with miRNA targets identified by miRanda algorithm [19-22]. 98 targets, which were found by both the integrated and the naïve model, and 31 targets, which 
Citation: Zhu J, Wu S, Yang J (2015) An Integrated Method for Detecting Micro RNA Target Proteins through Reverse-phase Protein Arrays. J Comput Sci Syst Biol 8: 012-033. doi:10.4172/jcsb. 1000166

Table 1: Table for the detailed point estimates of all unknown parameters and their standard error;Sample size was from 20 to 300 and the protein intensity located in the middle part of sigmoidal curve; the integrated model had a similar performance as the naïve model.

\begin{tabular}{|c|c|c|c|c|c|c|c|}
\hline & $\alpha_{1}$ & $\alpha_{2}$ & $\beta_{1}$ & $\boldsymbol{\beta}_{2}$ & $\boldsymbol{\beta}_{3}$ & $\sigma_{0}$ & $\sigma_{1}$ \\
\hline Sample size & 20 & & & & & & \\
\hline true value & 0 & 0 & 50 & 30000 & 1 & 1 & 500 \\
\hline naive model & $0.0213(0.0517)$ & $-0.0063(0.0117)$ & $78.13(22.56)$ & 29958.77 (31.48) & $1.0022(0.0014)$ & $0.9809(0.0053)$ & $463.61(1.34)$ \\
\hline integrated model & $0.015(0.052)$ & $-0.0062(0.0117)$ & $142.07(15.3)$ & $29881.73(28.8)$ & $1.0077(0.0014)$ & $0.9298(0.0051)$ & $490.89(1.24)$ \\
\hline true value & 0 & -0.1 & 50 & 30000 & 1 & 1 & 500 \\
\hline naive model & $-0.0062(0.0057)$ & $-0.1066(0.0117)$ & $78.43(22.69)$ & $29954.12(31.17)$ & $1.0023(0.0014)$ & $0.981(0.0053)$ & $463.75(1.34)$ \\
\hline integrated model & $-0.0106(0.0068)$ & $-0.1067(0.0117)$ & $136.82(15.25)$ & $29875.35(28.62)$ & $1.0078(0.0014)$ & $0.9299(0.005)$ & $490.51(1.23)$ \\
\hline true value & 0 & -0.3 & 50 & 30000 & 1 & 1 & 500 \\
\hline naive model & $-0.0015(0.0057)$ & $-0.3067(0.0117)$ & $83.04(23.4)$ & $29904.9(31.25)$ & $1.0044(0.0014)$ & $0.9811(0.0053)$ & 464.85 (1.38) \\
\hline integrated model & $-0.0126(0.0068)$ & $-0.3071(0.0117)$ & $168.89(15.35)$ & $29827.69(29.14)$ & $1.0102(0.0014)$ & $0.93(0.0051)$ & $491.49(1.23)$ \\
\hline true value & 0 & -0.5 & 50 & 30000 & 1 & 1 & 500 \\
\hline naive model & $-0.0015(0.0057)$ & $-0.5063(0.0117)$ & $81.23(22.74)$ & $29909.99(30.21)$ & $1.0045(0.0014)$ & $0.9807(0.0053)$ & $464.58(1.36)$ \\
\hline integrated model & $-0.0119(0.0068)$ & $-0.5069(0.0117)$ & $161.52(14.84)$ & $29838.19(28.02)$ & $1.0096(0.0014)$ & $0.9297(0.0051)$ & $491.18(1.23)$ \\
\hline true value & 0 & -1 & 50 & 30000 & 1 & 1 & 500 \\
\hline naive model & $-0.0043(0.0061)$ & $-1.0021(0.0117)$ & $23.03(20.93)$ & $30048.43(26.41)$ & $0.9997(0.0012)$ & $0.9795(0.0053)$ & $465.42(1.4)$ \\
\hline integrated model & $-0.0105(0.0068)$ & $-1.005(0.0117)$ & $91.21(13.22)$ & $29964.21(24.28)$ & $1.003(0.0012)$ & $0.9298(0.0051)$ & 491.06 (1.27) \\
\hline true value & 0 & -1.3 & 50 & 30000 & 1 & 1 & 500 \\
\hline naive model & $-0.0055(0.006)$ & $-1.3033(0.0117)$ & $39.67(18.69)$ & $30027.79(24.25)$ & $1.0018(0.0012)$ & $0.9792(0.0053)$ & $465.7(1.37)$ \\
\hline integrated model & $-0.0099(0.0067)$ & $-1.3077(0.0118)$ & $96.34(11.97)$ & $29952.63(22.46)$ & $1.0035(0.0011)$ & $0.9301(0.005)$ & $491.25(1.25)$ \\
\hline true value & 0 & -1.5 & 50 & 30000 & 1 & 1 & 500 \\
\hline naive model & $-0.0096(0.0063)$ & $-1.5032(0.0117)$ & $83.81(17.38)$ & $29973.69(22.32)$ & $1.0042(0.0011)$ & $0.9789(0.0053)$ & $464.47(1.35)$ \\
\hline integrated model & $-0.0111(0.0069)$ & $-1.5086(0.0117)$ & $124.24(11.18)$ & $29909.02(20.75)$ & $1.0054(0.0011)$ & $0.93(0.0051)$ & $491.11(1.28)$ \\
\hline Sample size & 50 & & & & & & \\
\hline true value & 0 & 0 & 50 & 30000 & 1 & 1 & 500 \\
\hline naive model & $-0.023(0.0283)$ & $0.0054(0.0064)$ & $-11.3(16.58)$ & $30137.24(20.17)$ & $0.9952(9 \mathrm{e}-04)$ & $0.9923(0.0032)$ & $457.55(0.85)$ \\
\hline integrated model & $-0.0259(0.0284)$ & $0.0053(0.0064)$ & $43.36(9.77)$ & $30057.24(18.46)$ & $0.9982(9 \mathrm{e}-04)$ & $0.9719(0.0032)$ & $495.97(0.79)$ \\
\hline true value & 0 & -0.1 & 50 & 30000 & 1 & 1 & 500 \\
\hline naive model & $-0.0012(0.0041)$ & $-0.0946(0.0064)$ & $-6.46(16.12)$ & $30141.23(19.69)$ & $0.9951(9 e-04)$ & $0.9925(0.0032)$ & $457.14(0.84)$ \\
\hline integrated model & $-0.0026(0.0043)$ & $-0.0947(0.0064)$ & $39.43(9.46)$ & $30061.21(18.05)$ & $0.9982(8 e-04)$ & $0.972(0.0032)$ & $495.79(0.79)$ \\
\hline true value & 0 & -0.3 & 50 & 30000 & 1 & 1 & 500 \\
\hline naive model & $0.0021(0.0041)$ & $-0.2942(0.0064)$ & $-14.38(15.88)$ & 30131.06 (18.59) & $0.9954(8 e-04)$ & $0.9923(0.0032)$ & $458.04(0.86)$ \\
\hline integrated model & $-9 e-04(0.0044)$ & $-0.2945(0.0064)$ & $36.9(8.99)$ & $30053.19(17.01)$ & $0.9983(8 e-04)$ & $0.9719(0.0032)$ & $496.54(0.78)$ \\
\hline true value & 0 & -0.5 & 50 & 30000 & 1 & 1 & 500 \\
\hline naive model & $0.0031(0.004)$ & $-0.4939(0.0064)$ & $-10.89(15.49)$ & $30113.24(17.77)$ & $0.9965(8 e-04)$ & $0.9919(0.0032)$ & $457(0.84)$ \\
\hline integrated model & $-0.002(0.0044)$ & $-0.4946(0.0064)$ & $46.42(8.56)$ & $30042.02(16.43)$ & $0.9988(8 e-04)$ & $0.9716(0.0032)$ & $496.05(0.79)$ \\
\hline true value & 0 & -1 & 50 & 30000 & 1 & 1 & 500 \\
\hline naive model & $-0.0031(0.0042)$ & $-0.9927(0.0064)$ & $16.45(13.86)$ & $30112.04(16.06)$ & $0.998(8 e-04)$ & $0.9915(0.0032)$ & $457.22(0.82)$ \\
\hline integrated model & $-0.0022(0.0044)$ & $-0.9948(0.0064)$ & $46.61(7.86)$ & $30043.54(14.75)$ & $0.9986(7 e-04)$ & $0.9719(0.0032)$ & $496.19(0.79)$ \\
\hline true value & 0 & -1.3 & 50 & 30000 & 1 & 1 & 500 \\
\hline naive model & $-0.0068(0.0043)$ & $-1.2911(0.0064)$ & $48.01(13.45)$ & 30080.17 (14.29) & $1.0002(7 e-04)$ & $0.9911(0.0032)$ & $457.21(0.82)$ \\
\hline integrated model & $-0.0018(0.0044)$ & $-1.2949(0.0064)$ & $60.83(7.18)$ & $30013.75(13.47)$ & $0.9997(7 e-04)$ & $0.9721(0.0032)$ & $496.15(0.79)$ \\
\hline true value & 0 & -1.5 & 50 & 30000 & 1 & 1 & 500 \\
\hline naive model & $-0.0078(0.0041)$ & $-1.4898(0.0064)$ & $77.09(11.46)$ & $30027.67(13.43)$ & $1.0032(7 e-04)$ & $0.9909(0.0032)$ & $456.69(0.78)$ \\
\hline integrated model & $-0.0026(0.0043)$ & $-1.4944(0.0064)$ & $82.21(6.6)$ & $29975.26(12.74)$ & $1.0017(7 \mathrm{e}-04)$ & $0.972(0.0032)$ & $496.5(0.77)$ \\
\hline Sample size & 100 & & & & & & \\
\hline true value & 0 & 0 & 50 & 30000 & 1 & 1 & 500 \\
\hline naive model & $0.0124(0.0207)$ & $-0.0032(0.0047)$ & $-21.78(12.82)$ & $30189.17(14.32)$ & $0.9927(6 e-04)$ & $0.997(0.0022)$ & $455.17(0.62)$ \\
\hline integrated model & $0.0143(0.0208)$ & $-0.0033(0.0047)$ & $7.17(6.74)$ & $30116.73(12.96)$ & $0.9951(6 e-04)$ & $0.9863(0.0022)$ & $498.45(0.57)$ \\
\hline true value & 0 & -0.1 & 50 & 30000 & 1 & 1 & 500 \\
\hline naive model & $0.0029(0.0032)$ & $-0.1028(0.0047)$ & $-46.45(12.49)$ & $30192.3(13.75)$ & $0.9929(6 e-04)$ & $0.9965(0.0022)$ & $454.27(0.6)$ \\
\hline integrated model & $-5 e-04(0.0032)$ & $-0.1029(0.0047)$ & $1.55(6.47)$ & $30125.41(12.4)$ & $0.9948(6 e-04)$ & $0.9859(0.0022)$ & $498.08(0.56)$ \\
\hline true value & 0 & -0.3 & 50 & 30000 & 1 & 1 & 500 \\
\hline naive model & $3 e-04(0.0033)$ & $-0.3033(0.0047)$ & $-26.94(12.46)$ & 30177.98 (13.77) & $0.9932(6 e-04)$ & $0.9968(0.0022)$ & $455.26(0.6)$ \\
\hline integrated model & $-9 e-04(0.0032)$ & $-0.3036(0.0047)$ & $11.3(6.47)$ & $30110.92(12.44)$ & $0.9951(6 e-04)$ & $0.9861(0.0022)$ & $498.77(0.56)$ \\
\hline true value & 0 & -0.5 & 50 & 30000 & 1 & 1 & 500 \\
\hline naive model & $0.0034(0.0033)$ & $-0.5024(0.0047)$ & $-29.46(12.41)$ & $30152.94(12.87)$ & $0.9948(6 e-04)$ & $0.9968(0.0022)$ & $454.68(0.59)$ \\
\hline
\end{tabular}


Citation: Zhu J, Wu S, Yang J (2015) An Integrated Method for Detecting Micro RNA Target Proteins through Reverse-phase Protein Arrays. J Comput Sci Syst Biol 8: 012-033. doi:10.4172/jcsb.1000166

\begin{tabular}{|c|c|c|c|c|c|c|c|}
\hline integrated model & $-0.002(0.0032)$ & $-0.5029(0.0047)$ & $23.79(6.41)$ & 30091.34 (11.81) & $0.996(6 e-04)$ & $0.9863(0.0022)$ & $498.68(0.56)$ \\
\hline true value & 0 & -1 & 50 & 30000 & 1 & 1 & 500 \\
\hline naive model & $-0.0063(0.0033)$ & $-1.0002(0.0047)$ & $18.58(10.56)$ & 30140.4 (11.11) & $0.9961(5 e-04)$ & $0.9965(0.0022)$ & $454.05(0.58)$ \\
\hline integrated model & $-0.0024(0.0032)$ & $-1.0018(0.0047)$ & 31.28 (5.38) & $30081.86(10.35)$ & $0.9964(5 \mathrm{e}-04)$ & $0.9864(0.0022)$ & $498.21(0.57)$ \\
\hline true value & 0 & -1.3 & 50 & 30000 & 1 & 1 & 500 \\
\hline naive model & $-0.0072(0.0033)$ & $-1.3008(0.0047)$ & $47.51(9.63)$ & 30088.26 (9.9) & $0.9992(5 e-04)$ & $0.9967(0.0022)$ & $453.78(0.59)$ \\
\hline integrated model & $-0.0024(0.0032)$ & $-1.3036(0.0047)$ & $52.33(4.87)$ & $30038.92(9.46)$ & $0.9982(5 e-04)$ & $0.9871(0.0022)$ & $498.32(0.59)$ \\
\hline true value & 0 & -1.5 & 50 & 30000 & 1 & 1 & 500 \\
\hline naive model & $-0.0066(0.0032)$ & $-1.5001(0.0047)$ & $65.64(8.32)$ & $30046.58(8.83)$ & $1.0018(4 \mathrm{e}-04)$ & $0.996(0.0022)$ & $452.58(0.56)$ \\
\hline integrated model & $-8 \mathrm{e}-04(0.0032)$ & $-1.5037(0.0047)$ & $62.17(4.51)$ & $30006.08(8.55)$ & $0.9998(4 \mathrm{e}-04)$ & $0.9864(0.0022)$ & $498.25(0.58)$ \\
\hline Sample size & 300 & & & & & & \\
\hline true value & 0 & 0 & 50 & 30000 & 1 & 1 & 500 \\
\hline naive model & $-0.0029(0.0118)$ & $-7 \mathrm{e}-04(0.0027)$ & $-7.69(8.36)$ & $30141.18(8.44)$ & $0.9945(4 \mathrm{e}-04)$ & $0.9998(0.0014)$ & $451.18(0.34)$ \\
\hline integrated model & $-0.0039(0.0118)$ & $-7 e-04(0.0027)$ & $17.74(3.87)$ & $30099.42(7.63)$ & $0.9956(4 e-04)$ & $0.9953(0.0014)$ & $499.45(0.32)$ \\
\hline true value & 0 & -0.1 & 50 & 30000 & 1 & 1 & 500 \\
\hline naive model & $3 e-04(0.0023)$ & $-0.1009(0.0026)$ & $-45.21(8.74)$ & $30156.77(7.84)$ & $0.9938(4 \mathrm{e}-04)$ & $0.9999(0.0014)$ & $451.88(0.34)$ \\
\hline integrated model & $-0.0084(0.002)$ & $-0.1009(0.0026)$ & $11.41(3.65)$ & $30117.72(7.15)$ & $0.9947(3 e-04)$ & $0.9955(0.0014)$ & $500(0.32)$ \\
\hline true value & 0 & -0.3 & 50 & 30000 & 1 & 1 & 500 \\
\hline naive model & $-0.0038(0.0024)$ & $-0.3005(0.0027)$ & $-19.8(8.81)$ & $30144.6(8.1)$ & $0.9945(4 \mathrm{e}-04)$ & $0.9996(0.0014)$ & $451.86(0.35)$ \\
\hline integrated model & $-0.0069(0.0019)$ & $-0.3006(0.0027)$ & $14.95(3.71)$ & $30101.39(7.38)$ & $0.9955(4 \mathrm{e}-04)$ & $0.9953(0.0014)$ & $499.85(0.31)$ \\
\hline true value & 0 & -0.5 & 50 & 30000 & 1 & 1 & 500 \\
\hline naive model & $-0.0041(0.0023)$ & $-0.5006(0.0026)$ & $-16.25(8.32)$ & $30142.07(7.35)$ & $0.9948(3 e-04)$ & $0.9996(0.0014)$ & $451.4(0.33)$ \\
\hline integrated model & $-0.0085(0.0019)$ & $-0.5009(0.0026)$ & $22.01(3.48)$ & $30102.75(6.82)$ & $0.9954(3 e-04)$ & $0.9953(0.0014)$ & $499.8(0.32)$ \\
\hline true value & 0 & -1 & 50 & 30000 & 1 & 1 & 500 \\
\hline naive model & $-0.0121(0.0023)$ & $-0.999(0.0027)$ & $30.28(7.06)$ & $30107.97(6.46)$ & $0.9968(3 e-04)$ & $0.9996(0.0014)$ & $451(0.33)$ \\
\hline integrated model & $-0.0082(0.002)$ & $-0.9999(0.0027)$ & $32.49(3.08)$ & $30070.72(6.22)$ & $0.9967(3 e-04)$ & $0.9955(0.0014)$ & $499.92(0.33)$ \\
\hline true value & 0 & -1.3 & 50 & 30000 & 1 & 1 & 500 \\
\hline naive model & $-0.0101(0.0021)$ & $-1.2999(0.0028)$ & $47.45(5.33)$ & 30058.55 (5.39) & $0.9997(3 e-04)$ & $1.0019(0.0027)$ & $448.96(0.31)$ \\
\hline integrated model & $-0.0077(0.002)$ & $-1.3008(0.0028)$ & $49.28(2.79)$ & $30036.58(5.22)$ & $0.9983(3 e-04)$ & $0.9955(0.0014)$ & $499.31(0.34)$ \\
\hline true value & 0 & -1.5 & 50 & 30000 & 1 & 1 & 500 \\
\hline naive model & $-0.0106(0.0021)$ & $-1.4962(0.0027)$ & $47.23(4.56)$ & $30059.42(5.23)$ & $1.0004(3 e-04)$ & $0.9997(0.0015)$ & $450.03(1.52)$ \\
\hline integrated model & $-0.0091(0.0021)$ & $-1.4986(0.0028)$ & $49.02(2.61)$ & $30043.6(5.15)$ & $0.9979(3 e-04)$ & $0.9955(0.0014)$ & $499.52(0.35)$ \\
\hline
\end{tabular}

Table 2: Table for the detailed point estimates of all unknown parameters and their standard error;Sample size was from 20 to $300 a n d$ the protein intensity located in the upper part of sigmoidal curve. Truncation was applied to the boundary of intensity level; the integrated model consistently yielded parameter estimates of $\boldsymbol{\alpha}_{2}$ with similar or much less standard errors than the naïve model.

\begin{tabular}{|c|c|c|c|c|c|c|c|}
\hline & $\alpha_{1}$ & $\alpha_{2}$ & $\beta_{1}$ & $\boldsymbol{\beta}_{2}$ & $\boldsymbol{\beta}_{3}$ & $\sigma_{0}$ & $\sigma_{1}$ \\
\hline Sample size & 20 & & & & & & \\
\hline true value & 5 & 0 & 50 & 30000 & 1 & 1 & 500 \\
\hline naive model & $2.3538(0.0337)$ & $0.0108(0.0348)$ & 21673.99 (73.67) & $8063.19(72.22)$ & $1.8432(0.0062)$ & $1.5515(0.1377)$ & $505.9(4.52)$ \\
\hline integrated model & $4.4653(0.08)$ & $-0.0161(0.0139)$ & $-274276.76(52197.04)$ & $304133.74(52200.42)$ & $1.2805(0.011)$ & $0.9099(0.0111)$ & 457.39 (1.95) \\
\hline true value & 5 & -0.1 & 50 & 30000 & 1 & 1 & 500 \\
\hline naive model & $2.3656(0.0399)$ & $-0.0895(0.0592)$ & $21700.84(71.7)$ & $8041.22(70.25)$ & $1.8401(0.0059)$ & $1.6134(0.1588)$ & $504.84(3.24)$ \\
\hline integrated model & $4.5686(0.0859)$ & $-0.0693(0.0418)$ & $-330800.61(56594.51)$ & 360639.78 (56591.54) & $1.2918(0.0288)$ & $0.9732(0.0742)$ & $458.96(2)$ \\
\hline true value & 5 & -0.3 & 50 & 30000 & 1 & 1 & 500 \\
\hline naive model & $2.3695(0.0275)$ & $-0.3577(0.0482)$ & $21516.03(74.6)$ & $8221.13(73.15)$ & $1.8385(0.0057)$ & $1.5135(0.1068)$ & $505.61(3.97)$ \\
\hline integrated model & $4.403(0.0769)$ & $-0.308(0.0123)$ & $-249677.33(50408.57)$ & $279512.95(50405.41)$ & $1.2762(0.0105)$ & $0.8937(0.0099)$ & $456.25(1.72)$ \\
\hline true value & 5 & -0.5 & 50 & 30000 & 1 & 1 & 500 \\
\hline naive model & $2.4446(0.0374)$ & $-0.7107(0.0782)$ & 21269.05 (74.86) & 8464.35 (73.51) & $1.8481(0.0059)$ & $1.6564(0.1521)$ & $507.56(2.77)$ \\
\hline integrated model & $4.5227(0.0783)$ & $-0.5035(0.0123)$ & $-250395.86(48590.31)$ & $280258.79(48592.42)$ & $1.2486(0.0097)$ & $0.8891(0.0102)$ & $457.35(1.82)$ \\
\hline true value & 5 & -1 & 50 & 30000 & 1 & 1 & 500 \\
\hline naive model & $2.7712(0.0505)$ & $-1.4615(0.1208)$ & $19902.57(90.02)$ & $9814.9(88.76)$ & $1.8388(0.0058)$ & $2.1685(0.1959)$ & $527.34(4.87)$ \\
\hline integrated model & $4.1187(0.0636)$ & $-1.0024(0.0148)$ & $-52274.05(21283.7)$ & 82100.65 (21281.33) & $1.2984(0.0263)$ & $0.8417(0.0365)$ & $469.74(2.81)$ \\
\hline true value & 5 & -1.3 & 50 & 30000 & 1 & 1 & 500 \\
\hline naive model & $2.9411(0.0548)$ & $-1.7328(0.0838)$ & 18770.02 (98.58) & 10936.33 (97.49) & $1.828(0.0054)$ & $2.2838(0.2277)$ & $539.5(4.31)$ \\
\hline integrated model & $4.1799(0.0528)$ & $-1.3159(0.0145)$ & $-81574.65(30566.75)$ & $111394.19(30567.8)$ & $1.2845(0.0095)$ & $0.8634(0.0125)$ & 478.65 (2.39) \\
\hline true value & 5 & -1.5 & 50 & 30000 & 1 & 1 & 500 \\
\hline naive model & $3.2206(0.0717)$ & $-2.3778(0.1593)$ & $18002.82(105.51)$ & $11696.6(104.59)$ & $1.8231(0.0057)$ & $2.9557(0.2621)$ & $560.41(7.25)$ \\
\hline integrated model & $3.6493(0.6203)$ & $-0.964(0.6069)$ & $-51974.4(22391.12)$ & 81776.04 (22386.56) & $1.2932(0.0201)$ & $0.8548(0.0355)$ & $488.64(3.71)$ \\
\hline Sample size & 50 & & & & & & \\
\hline true value & 5 & 0 & 50 & 30000 & 1 & 1 & 500 \\
\hline
\end{tabular}


Citation: Zhu J, Wu S, Yang J (2015) An Integrated Method for Detecting Micro RNA Target Proteins through Reverse-phase Protein Arrays. J Comput Sci Syst Biol 8: 012-033. doi:10.4172/jcsb.1000166

\begin{tabular}{|c|c|c|c|c|c|c|c|}
\hline naive model & $2.7437(0.0369)$ & $-0.0469(0.0526)$ & 20499.39 (63.98) & $9225.41(62.87)$ & $1.7402(0.0037)$ & $2.7635(0.2377)$ & $485.25(2.14)$ \\
\hline integrated model & $3.5198(0.0405)$ & $0.0089(0.0067)$ & 8598.57 (1581.12) & $21172.62(1582.55)$ & $1.4285(0.0103)$ & $0.8595(0.0138)$ & $489.77(1.59)$ \\
\hline true value & 5 & -0.1 & 50 & 30000 & 1 & 1 & 500 \\
\hline naive model & $2.7316(0.0347)$ & $-0.1643(0.0384)$ & $20463.66(65.62)$ & $9261.85(64.57)$ & $1.738(0.0038)$ & $2.6338(0.2266)$ & $484.29(1.72)$ \\
\hline integrated model & $3.4811(0.0385)$ & $-0.0898(0.0067)$ & $9927.86(1621.29)$ & $19842.78(1622.67)$ & $1.434(0.0104)$ & $0.8728(0.0129)$ & $491.19(1.61)$ \\
\hline true value & 5 & -0.3 & 50 & 30000 & 1 & 1 & 500 \\
\hline naive model & $2.8069(0.0401)$ & $-0.4472(0.047)$ & $20293.49(66.02)$ & $9426.7(65.05)$ & $1.7416(0.0037)$ & $2.9565(0.2576)$ & $486.58(1.97)$ \\
\hline integrated model & $3.4894(0.0357)$ & $-0.2882(0.0067)$ & 12135.89 (534.51) & $17635.35(537.21)$ & $1.4246(0.0101)$ & $0.8701(0.0131)$ & $491.18(1.66)$ \\
\hline true value & 5 & -0.5 & 50 & 30000 & 1 & 1 & 500 \\
\hline naive model & $2.828(0.0387)$ & $-0.6441(0.0451)$ & $19946.91(69.44)$ & $9770.55(68.46)$ & $1.7402(0.0038)$ & $2.8163(0.2509)$ & $491.23(2.77)$ \\
\hline integrated model & $3.5327(0.0365)$ & $-0.4769(0.0067)$ & $11365.82(735.99)$ & $18404.38(738.2)$ & $1.4188(0.01)$ & $0.8762(0.0124)$ & $493.4(1.7)$ \\
\hline true value & 5 & -1 & 50 & 30000 & 1 & 1 & 500 \\
\hline naive model & $3.2183(0.047)$ & $-1.7113(0.0905)$ & $18367.63(79.4)$ & $11331.51(78.56)$ & $1.7405(0.0037)$ & $3.9333(0.301)$ & $506.65(2.69)$ \\
\hline integrated model & $3.4259(0.029)$ & $-0.9716(0.0072)$ & $13477.54(288.17)$ & $16260.6(291.47)$ & $1.4497(0.0086)$ & $0.7908(0.0171)$ & $512.56(1.84)$ \\
\hline true value & 5 & -1.3 & 50 & 30000 & 1 & 1 & 500 \\
\hline naive model & $3.5889(0.0593)$ & $-2.5651(0.1361)$ & $17174.96(86.16)$ & $12514.49(85.49)$ & $1.7375(0.0037)$ & $5.4373(0.3774)$ & $521.52(3.13)$ \\
\hline integrated model & $3.2866(0.1729)$ & $-1.4043(0.1428)$ & $13337.94(260.58)$ & $16383.67(263.67)$ & $1.4403(0.0146)$ & $0.8708(0.1229)$ & $528.37(2.55)$ \\
\hline true value & 5 & -1.5 & 50 & 30000 & 1 & 1 & 500 \\
\hline naive model & $3.8296(0.0621)$ & $-2.9831(0.1416)$ & $16141.61(89.86)$ & $13546.24(89.49)$ & $1.7198(0.0039)$ & $6.1208(0.3784)$ & $523.94(2.07)$ \\
\hline integrated model & $3.305(0.1669)$ & $-1.3648(0.105)$ & $13436.45(198.21)$ & $16273.32(201.3)$ & $1.4225(0.0332)$ & $0.9029(0.1719)$ & $536.53(2.56)$ \\
\hline Sample size & 100 & & & & & & \\
\hline true value & 5 & 0 & 50 & 30000 & 1 & 1 & 500 \\
\hline naive model & $2.9087(0.0269)$ & $-0.039(0.0434)$ & $19661.6(56.83)$ & $10058.46(56.2)$ & $1.6748(0.003)$ & $3.337(0.2366)$ & $468.53(1.22)$ \\
\hline integrated model & $3.0493(0.0261)$ & $-0.0055(0.006)$ & $16387.53(641.05)$ & $13333.07(642.19)$ & $1.5292(0.0075)$ & $0.8232(0.0178)$ & $501.83(1.1)$ \\
\hline true value & 5 & -0.1 & 50 & 30000 & 1 & 1 & 500 \\
\hline naive model & $2.9097(0.0291)$ & $-0.1508(0.043)$ & $19584.67(57.9)$ & $10137.22(57.29)$ & $1.6675(0.0028)$ & $3.2625(0.2545)$ & $469.76(1.53)$ \\
\hline integrated model & $3.0247(0.0234)$ & $-0.0985(0.0065)$ & $17062.36(191.54)$ & $12657.31(194.2)$ & $1.5329(0.0073)$ & $0.8748(0.0133)$ & $502.86(1.06)$ \\
\hline true value & 5 & -0.3 & 50 & 30000 & 1 & 1 & 500 \\
\hline naive model & $2.9106(0.0275)$ & $-0.3621(0.0361)$ & $19313.04(62.78)$ & $10404.89(62.09)$ & $1.6712(0.003)$ & $3.0649(0.2302)$ & $469.78(0.91)$ \\
\hline integrated model & $3.0442(0.0224)$ & $-0.3021(0.0073)$ & $16981.12(176.62)$ & $12738.09(179.23)$ & $1.5236(0.007)$ & $0.8185(0.0172)$ & $504.55(1.09)$ \\
\hline true value & 5 & -0.5 & 50 & 30000 & 1 & 1 & 500 \\
\hline naive model & $3.0186(0.0323)$ & $-0.7679(0.044)$ & $18919.14(64.29)$ & $10793.17(63.66)$ & $1.674(0.003)$ & $3.701(0.2824)$ & $474.8(1.61)$ \\
\hline integrated model & $3.0784(0.0226)$ & $-0.4853(0.0049)$ & $16620.4(198.41)$ & $13095.57(200.87)$ & $1.5193(0.0068)$ & $0.8203(0.0165)$ & $508.61(1.12)$ \\
\hline true value & 5 & -1 & 50 & 30000 & 1 & 1 & 500 \\
\hline naive model & $3.5281(0.0442)$ & $-2.0565(0.0999)$ & $17213.8(74.03)$ & $12482.15(73.63)$ & $1.6745(0.003)$ & $5.9013(0.3507)$ & $486.43(0.92)$ \\
\hline integrated model & $3.1546(0.0163)$ & $-0.9644(0.0055)$ & $16182.07(131.53)$ & $13518.68(133.43)$ & $1.5056(0.0053)$ & $0.7447(0.0202)$ & $521.9(1.12)$ \\
\hline true value & 5 & -1.3 & 50 & 30000 & 1 & 1 & 500 \\
\hline naive model & $3.8155(0.0476)$ & $-2.6141(0.0999)$ & $15813.29(80.09)$ & $13875.57(79.85)$ & $1.6668(0.0034)$ & $7.1509(0.3873)$ & $504.16(2.87)$ \\
\hline integrated model & $3.28(0.0142)$ & $-1.2628(0.0074)$ & $15230.07(110.54)$ & 14467.41 (111.99) & $1.4792(0.0047)$ & $0.7278(0.0212)$ & $531.28(1.16)$ \\
\hline true value & 5 & -1.5 & 50 & 30000 & 1 & 1 & 500 \\
\hline naive model & $4.23(0.0626)$ & $-3.6045(0.1408)$ & $14844.17(83.8)$ & $14844.17(83.59)$ & $1.6571(0.0034)$ & $9.335(0.4778)$ & $513.6(2.09)$ \\
\hline integrated model & $3.4028(0.016)$ & $-1.4779(0.0122)$ & $14282.26(116.92)$ & $15415.65(118.47)$ & $1.4536(0.0046)$ & $0.6573(0.0252)$ & $539.12(1.27)$ \\
\hline Sample size & 300 & & & & & & \\
\hline true value & 5 & 0 & 50 & 30000 & 1 & 1 & 500 \\
\hline naive model & $3.0135(0.0208)$ & $0.0285(0.0232)$ & $18437.53(67.92)$ & $11288.37(67.65)$ & $1.5908(0.003)$ & $3.2476(0.263)$ & $451.57(1.21)$ \\
\hline integrated model & $2.9(0.0112)$ & $5 e-04(0.0039)$ & $18348.42(75.58)$ & $11365.95(76.43)$ & $1.5243(0.0043)$ & $0.8799(0.0187)$ & $498.43(0.68)$ \\
\hline true value & 5 & -0.1 & 50 & 30000 & 1 & 1 & 500 \\
\hline naive model & $3.0361(0.0228)$ & $-0.1808(0.0329)$ & $18456.78(69.96)$ & $11268.42(69.74)$ & $1.592(0.0032)$ & $3.577(0.2943)$ & $454.1(2.08)$ \\
\hline integrated model & $2.9366(0.0163)$ & $-0.0989(0.0074)$ & $18113.81(121.05)$ & $11603.85(122.77)$ & $1.516(0.0054)$ & $0.879(0.0201)$ & $496.92(0.77)$ \\
\hline true value & 5 & -0.3 & 50 & 30000 & 1 & 1 & 500 \\
\hline naive model & $3.0804(0.027)$ & $-0.3768(0.0336)$ & $18175.54(74.23)$ & $11545.95(73.97)$ & $1.5939(0.0032)$ & $4.1281(0.3787)$ & $453.35(1.29)$ \\
\hline integrated model & $2.9395(0.0132)$ & $-0.2858(0.004)$ & $18058.62(94.19)$ & $11655.43(95.21)$ & $1.5166(0.0046)$ & $0.8667(0.0213)$ & $498.87(0.69)$ \\
\hline true value & 5 & -0.5 & 50 & 30000 & 1 & 1 & 500 \\
\hline naive model & $3.1502(0.0254)$ & $-0.7234(0.0412)$ & $17717.61(82.68)$ & $11998.41(82.46)$ & $1.5974(0.0034)$ & $3.9477(0.3312)$ & $454.13(0.75)$ \\
\hline integrated model & $2.9937(0.0129)$ & $-0.4811(0.0042)$ & $17680.24(87.96)$ & $12031.57(88.86)$ & $1.5063(0.0047)$ & $0.8291(0.0251)$ & $500.36(0.78)$ \\
\hline true value & 5 & -1 & 50 & 30000 & 1 & 1 & 500 \\
\hline naive model & $3.5669(0.043)$ & $-1.7535(0.09)$ & $15740.25(113.86)$ & $13964.36(113.86)$ & $1.5926(0.0043)$ & $6.4576(0.5025)$ & $471.3(3.33)$ \\
\hline integrated model & $3.2607(0.0177)$ & $-0.9702(0.0074)$ & $15650.42(130.59)$ & $14066.12(132.08)$ & $1.4461(0.0057)$ & $0.8718(0.0259)$ & $505.15(0.98)$ \\
\hline true value & 5 & -1.3 & 50 & 30000 & 1 & 1 & 500 \\
\hline naive model & $4.0045(0.1514)$ & $-2.2565(0.5547)$ & $13772.02(120.38)$ & $15929.25(120.41)$ & $1.5632(0.0045)$ & $12.2208(2.356)$ & $480.58(3.11)$ \\
\hline integrated model & $3.1969(0.3036)$ & $-0.7316(0.5495)$ & $13720.47(126.43)$ & $16004.76(128)$ & $1.3919(0.0097)$ & $2.9218(2.2545)$ & $514.38(4.56)$ \\
\hline true value & 5 & -1.5 & 50 & 30000 & 1 & 1 & 500 \\
\hline naive model & $4.4603(0.0723)$ & $-3.707(0.1756)$ & $12633.68(117.4)$ & $17067.83(117.75)$ & $1.5489(0.0043)$ & $12.0708(0.7155)$ & $485.18(1.18)$ \\
\hline integrated model & $3.6474(0.0193)$ & $-1.4779(0.0129)$ & $12443.71(145.16)$ & $17289.63(147.48)$ & $1.3512(0.0055)$ & $0.6374(0.0466)$ & $510.48(1.31)$ \\
\hline
\end{tabular}


Citation: Zhu J, Wu S, Yang J (2015) An Integrated Method for Detecting Micro RNA Target Proteins through Reverse-phase Protein Arrays. J Comput Sci Syst Biol 8: 012-033. doi:10.4172/jcsb.1000166

Table 3: A list of miRNA/protein pairs suggested by the naïve model and the integrated model and they were classed into three groups: "Found by the integrated model only", "Found by both the integrated model and the naïve model" and "Found by the naïve model only". Pairs found by the integrated model were sorted by ascending order of adjusted p-values from the integrated model and the naïve model, respectively. Pairs found by the naïve model only were sorted by ascending order of adjusted p-values from the naïve model andintegrated model, respectively.In addition, a number "1" will mark under the column for pairs found by MirTarbase, MirTarBase with strong experimental evidences or miRanda, and those pairs will be list on the top of each group after pairs got ordered.

\begin{tabular}{|c|c|c|c|c|c|c|c|}
\hline Composite Element REF & miRNA & \multicolumn{3}{|c|}{ Corresponding genes } & MirTarbase & $\begin{array}{l}\text { MirTarbase (Supported by } \\
\text { strong experimental evidences) }\end{array}$ & miRanda \\
\hline \multicolumn{8}{|l|}{ Found by the integrated model only } \\
\hline p53-r-v & hsa-mir-605 & TP53 & & & 1 & 1 & \\
\hline$n$-cadherin-r-v & hsa-mir-511-2 & $\mathrm{CDH} 2$ & & & & & 1 \\
\hline akt-r-v & hsa-mir-511-1 & AKT1 & AKT2 & AKT3 & & & 1 \\
\hline eef2k-r-v & hsa-mir-486 & EEF2K & & & & & 1 \\
\hline p53-r-v & hsa-mir-181a-1 & TP53 & & & & & 1 \\
\hline smad4-m-c & hsa-mir-142 & SMAD4 & & & & & 1 \\
\hline c-met-m-c & hsa-mir-223 & MET & & & & & 1 \\
\hline caspase-8-m-c & hsa-mir-541 & CASP8 & & & & & 1 \\
\hline pcna-m-v & hsa-mir-223 & PCNA & & & & & 1 \\
\hline$n$-cadherin-r-v & hsa-mir-146a & $\mathrm{CDH} 2$ & & & & & 1 \\
\hline syk-m-v & hsa-mir-369 & SYK & & & & & 1 \\
\hline alpha-catenin-m-v & hsa-mir-22 & CTNNA1 & & & & & 1 \\
\hline smad3-r-v & hsa-mir-142 & SMAD3 & & & & & 1 \\
\hline chk1-r-v & hsa-mir-605 & CHEK1 & & & & & 1 \\
\hline er-alpha-r-v & hsa-mir-181a-2 & ESR1 & & & & & 1 \\
\hline aib1-m-v & hsa-mir-605 & NCOA3 & & & & & 1 \\
\hline rad50-m-c & hsa-mir-22 & RAD50 & & & & & 1 \\
\hline caspase-8-m-c & hsa-mir-483 & CASP8 & & & & & 1 \\
\hline syk-m-v & hsa-mir-193a & SYK & & & & & 1 \\
\hline jnk2-r-c & hsa-mir-511-1 & MAPK9 & & & & & 1 \\
\hline p53-r-v & hsa-mir-588 & TP53 & & & & & 1 \\
\hline dj-1-r-c & hsa-mir-145 & PARK7 & & & & & 1 \\
\hline akt-r-v & hsa-mir-142 & AKT1 & AKT2 & AKT3 & & & 1 \\
\hline p27-r-v & hsa-mir-205 & CDKN1B & & & & & 1 \\
\hline beta-catenin-r-v & hsa-mir-485 & CTNNB1 & & & & & 1 \\
\hline c-kit-r-v & hsa-mir-21 & KIT & & & & & 1 \\
\hline chk1-r-v & hsa-let-7b & CHEK1 & & & & & 1 \\
\hline b-raf-m-na & hsa-mir-511-1 & BRAF & & & & & 1 \\
\hline stat5-alpha-r-v & hsa-mir-1224 & STAT5A & & & & & 1 \\
\hline igf-1r-beta-r-c & hsa-let-7b & IGF1R & & & & & 1 \\
\hline pea-15-r-v & hsa-mir-541 & PEA15 & & & & & 1 \\
\hline beta-catenin-r-v & hsa-mir-1228 & CTNNB1 & & & & & 1 \\
\hline c-raf-r-v & hsa-mir-1295 & RAF1 & & & & & \\
\hline msh2-m-c & hsa-mir-1247 & $\mathrm{MSH} 2$ & & & & & \\
\hline msh2-m-c & hsa-mir-199a-1 & $\mathrm{MSH} 2$ & & & & & \\
\hline aib1-m-v & hsa-mir-150 & NCOA3 & & & & & \\
\hline bcl-2-m-v & hsa-mir-1307 & BCL2 & & & & & \\
\hline alpha-catenin-m-v & hsa-mir-511-2 & CTNNA1 & & & & & \\
\hline alpha-catenin-m-v & hsa-mir-511-1 & CTNNA1 & & & & & \\
\hline aib1-m-v & hsa-mir-214 & NCOA3 & & & & & \\
\hline bcl-2-m-v & hsa-mir-652 & BCL2 & & & & & \\
\hline ar-r-v & hsa-mir-224 & AR & & & & & \\
\hline k-ras-m-c & hsa-mir-150 & KRAS & & & & & \\
\hline ciap-r-v & hsa-mir-223 & BIRC2 & & & & & \\
\hline ciap-r-v & hsa-mir-140 & $\mathrm{BIRC2}$ & & & & & \\
\hline ciap-r-v & hsa-mir-511-2 & BIRC2 & & & & & \\
\hline aib1-m-v & hsa-mir-1228 & NCOA3 & & & & & \\
\hline claudin-7-r-v & hsa-mir-140 & CLDN7 & & & & & \\
\hline ar-r-v & hsa-mir-1228 & AR & & & & & \\
\hline bcl-xl-r-c & hsa-mir-1295 & BCL2L1 & & & & & \\
\hline ar-r-v & hsa-mir-511-1 & AR & & & & & \\
\hline eef2k-r-v & hsa-mir-181a-2 & EEF2K & & & & & \\
\hline tau-m-c & hsa-mir-142 & MAPT & & & & & \\
\hline cdk1-r-v & hsa-mir-1247 & CDC2 & & & & & \\
\hline
\end{tabular}


Citation: Zhu J, Wu S, Yang J (2015) An Integrated Method for Detecting Micro RNA Target Proteins through Reverse-phase Protein Arrays. J Comput Sci Syst Biol 8: 012-033. doi:10.4172/jcsb.1000166

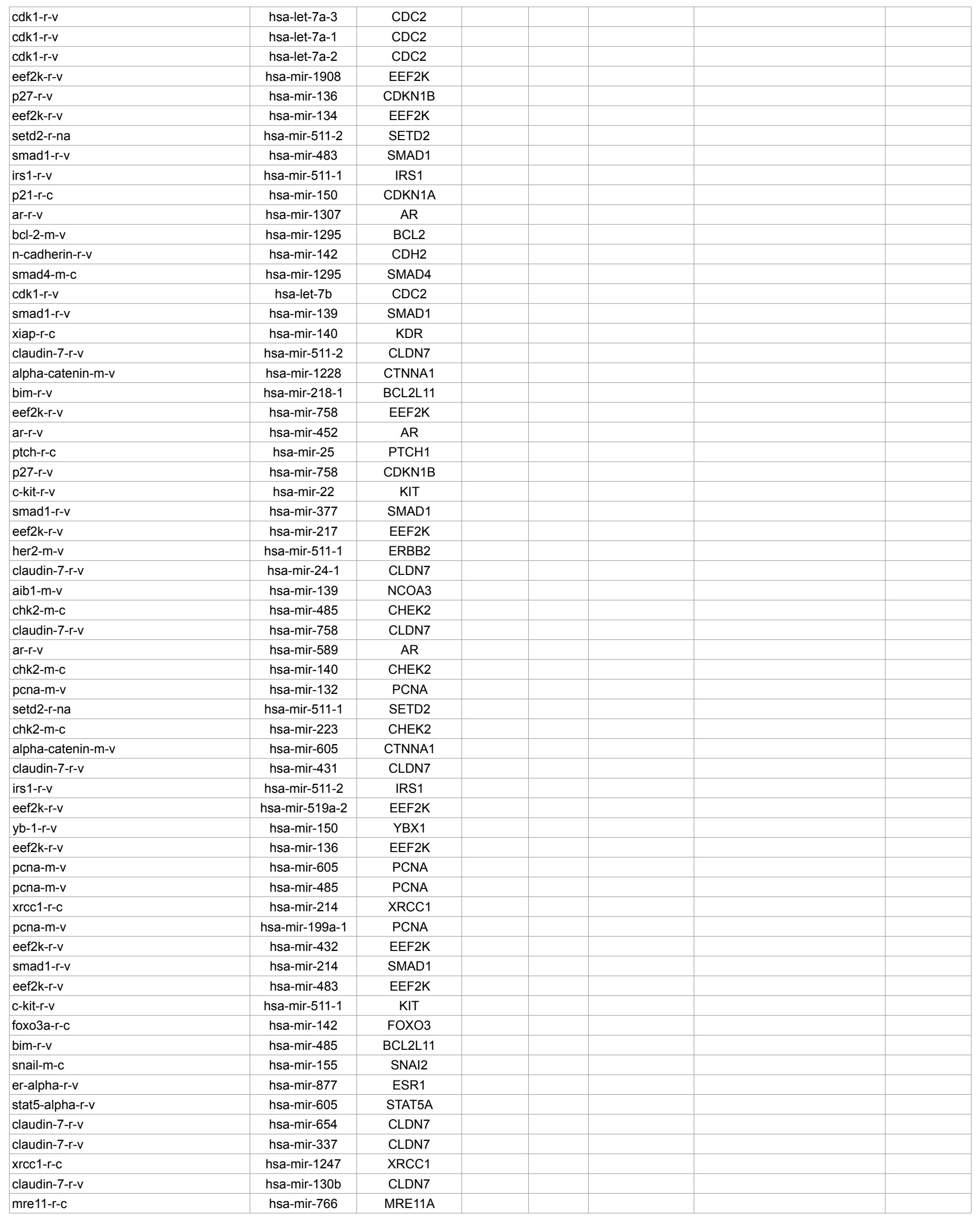


Citation: Zhu J, Wu S, Yang J (2015) An Integrated Method for Detecting Micro RNA Target Proteins through Reverse-phase Protein Arrays. J Comput Sci Syst Biol 8: 012-033. doi:10.4172/jcsb.1000166

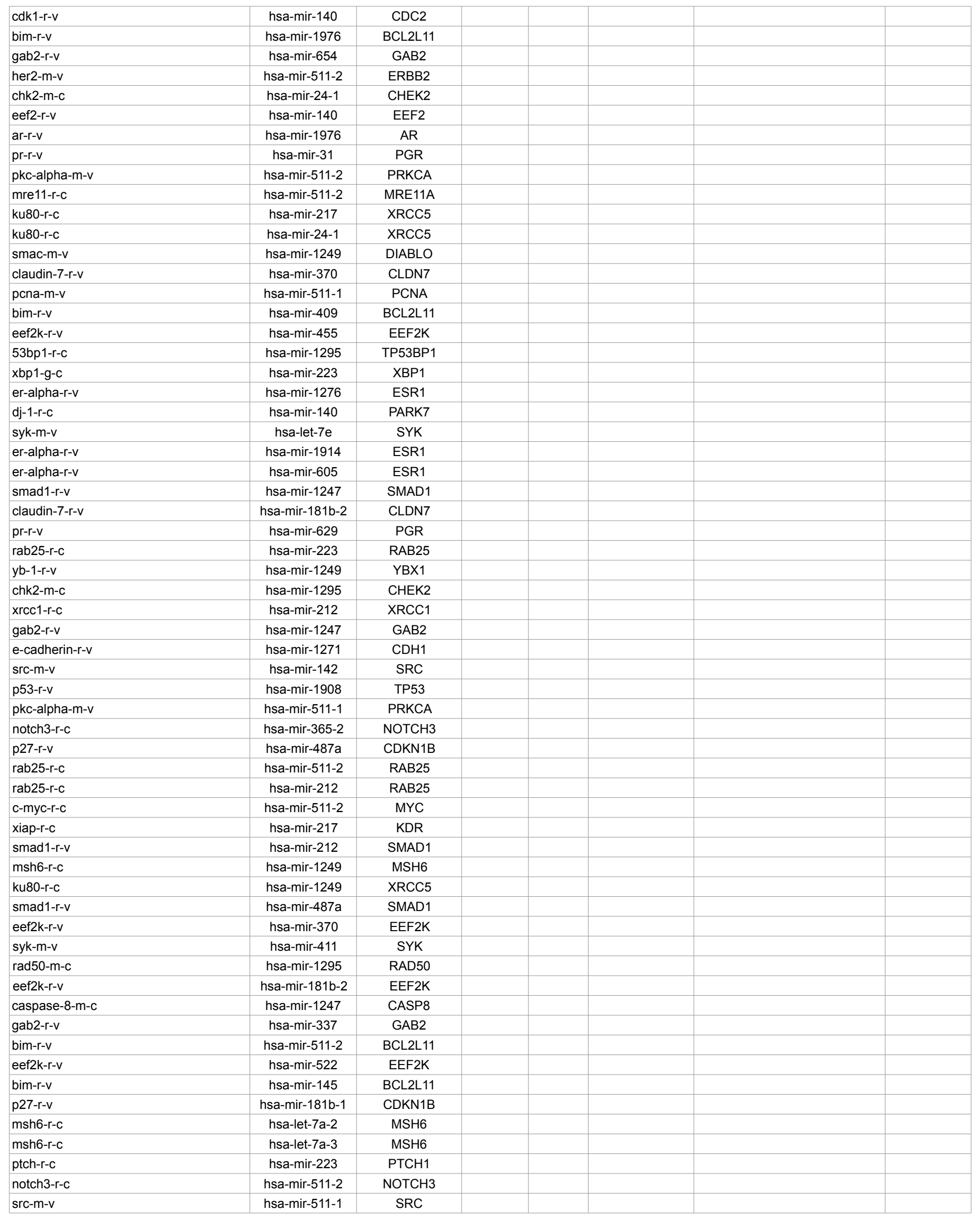


Citation: Zhu J, Wu S, Yang J (2015) An Integrated Method for Detecting Micro RNA Target Proteins through Reverse-phase Protein Arrays. J Comput Sci Syst Biol 8: 012-033. doi:10.4172/jcsb.1000166

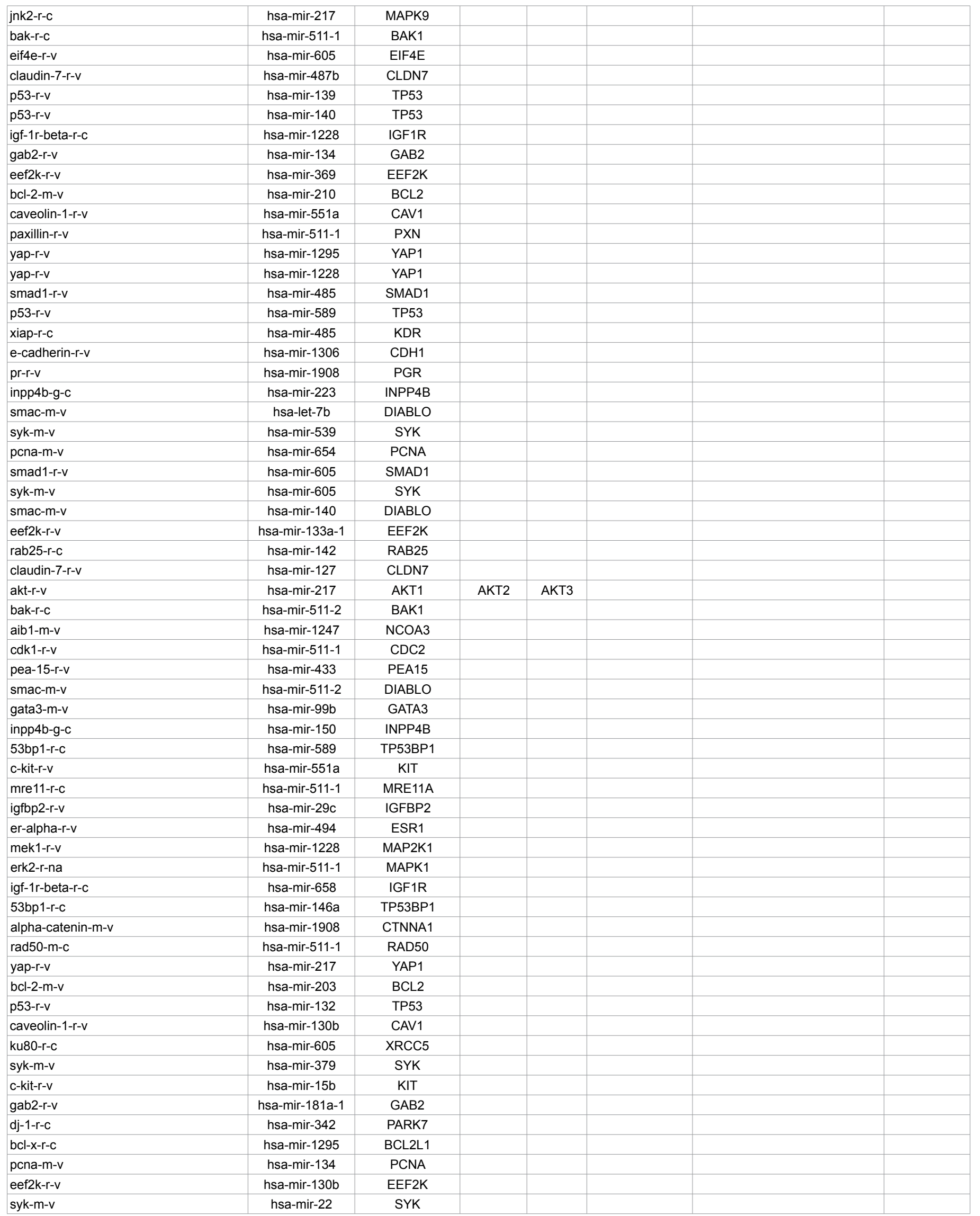


Citation: Zhu J, Wu S, Yang J (2015) An Integrated Method for Detecting Micro RNA Target Proteins through Reverse-phase Protein Arrays. J Comput Sci Syst Biol 8: 012-033. doi:10.4172/jcsb.1000166

\begin{tabular}{|c|c|c|c|c|c|}
\hline igf-1r-beta-r-c & hsa-mir-203 & IGF1R & & & \\
\hline paxillin-r-v & hsa-mir-766 & $\mathrm{PXN}$ & & & \\
\hline e-cadherin-r-v & hsa-mir-329-1 & $\mathrm{CDH} 1$ & & & \\
\hline pcna-m-v & hsa-mir-493 & PCNA & & & \\
\hline smac-m-v & hsa-mir-588 & DIABLO & & & \\
\hline smad1-r-v & hsa-mir-455 & SMAD1 & & & \\
\hline rad50-m-c & hsa-mir-1247 & RAD50 & & & \\
\hline inpp4b-g-c & hsa-mir-766 & INPP4B & & & \\
\hline p70s6k-r-v & hsa-mir-1247 & RPS6KB1 & & & \\
\hline cdk1-r-v & hsa-mir-605 & CDC2 & & & \\
\hline smac-m-v & hsa-mir-605 & DIABLO & & & \\
\hline smad1-r-v & hsa-mir-1228 & SMAD1 & & & \\
\hline alpha-catenin-m-v & hsa-mir-214 & CTNNA1 & & & \\
\hline \multicolumn{6}{|c|}{ Found by both the integrated model and the naïve model } \\
\hline notch3-r-c & hsa-mir-150 & NOTCH3 & 1 & 1 & 1 \\
\hline er-alpha-r-v & hsa-mir-18a & ESR1 & 1 & 1 & 1 \\
\hline p53-r-v & hsa-mir-150 & TP53 & 1 & 1 & \\
\hline beta-catenin-r-v & hsa-mir-214 & CTNNB1 & 1 & 1 & \\
\hline bim-r-v & hsa-mir-181a-1 & BCL2L11 & 1 & 1 & \\
\hline igf-1r-beta-r-c & hsa-mir-223 & IGF1R & 1 & 1 & \\
\hline igf-1r-beta-r-c & hsa-mir-139 & IGF1R & 1 & 1 & \\
\hline p27-r-v & hsa-mir-181a-1 & CDKN1B & 1 & 1 & \\
\hline igf-1r-beta-r-c & hsa-mir-145 & IGF1R & 1 & 1 & \\
\hline smad3-r-v & hsa-mir-155 & SMAD3 & 1 & 1 & \\
\hline msh6-r-c & hsa-mir-21 & MSH6 & 1 & 1 & \\
\hline caveolin-1-r-v & hsa-mir-7-1 & CAV1 & 1 & & 1 \\
\hline bim-r-v & hsa-let-7a-2 & BCL2L11 & 1 & & 1 \\
\hline bim-r-v & hsa-let-7a-1 & BCL2L11 & 1 & & 1 \\
\hline bim-r-v & hsa-let-7a-3 & BCL2L11 & 1 & & 1 \\
\hline$n$-cadherin-r-v & hsa-mir-150 & $\mathrm{CDH} 2$ & & & 1 \\
\hline yap-r-v & hsa-mir-150 & YAP1 & & & 1 \\
\hline er-alpha-r-v & hsa-mir-766 & ESR1 & & & 1 \\
\hline ku80-r-c & hsa-mir-223 & XRCC5 & & & 1 \\
\hline beta-catenin-r-v & hsa-mir-223 & CTNNB1 & & & 1 \\
\hline er-alpha-r-v & hsa-mir-493 & ESR1 & & & 1 \\
\hline claudin-7-r-v & hsa-mir-214 & CLDN7 & & & 1 \\
\hline e-cadherin-r-v & hsa-mir-605 & $\mathrm{CDH} 1$ & & & 1 \\
\hline claudin-7-r-v & hsa-mir-1228 & CLDN7 & & & 1 \\
\hline beta-catenin-r-v & hsa-mir-511-1 & CTNNB1 & & & 1 \\
\hline er-alpha-r-v & hsa-mir-337 & ESR1 & & & 1 \\
\hline er-alpha-r-v & hsa-mir-299 & ESR1 & & & 1 \\
\hline bim-r-v & hsa-let-7b & BCL2L11 & & & 1 \\
\hline syk-m-v & hsa-mir-409 & SYK & & & 1 \\
\hline igf-1r-beta-r-c & hsa-mir-142 & IGF1R & & & 1 \\
\hline e-cadherin-r-v & hsa-mir-130b & $\mathrm{CDH} 1$ & & & 1 \\
\hline pcna-m-v & hsa-let-7a-2 & PCNA & & & 1 \\
\hline beta-catenin-r-v & hsa-mir-511-2 & CTNNB1 & & & 1 \\
\hline syk-m-v & hsa-mir-654 & SYK & & & 1 \\
\hline e-cadherin-r-v & hsa-mir-493 & $\mathrm{CDH} 1$ & & & 1 \\
\hline eef2k-r-v & hsa-mir-605 & EEF2K & & & 1 \\
\hline syk-m-v & hsa-mir-337 & SYK & & & 1 \\
\hline snail-m-c & hsa-mir-150 & SNAI2 & & & 1 \\
\hline igf-1r-beta-r-c & hsa-mir-511-1 & IGF1R & & & 1 \\
\hline e-cadherin-r-v & hsa-mir-22 & $\mathrm{CDH} 1$ & & & 1 \\
\hline er-alpha-r-v & hsa-mir-323 & ESR1 & & & 1 \\
\hline er-alpha-r-v & hsa-mir-411 & ESR1 & & & 1 \\
\hline bcl-2-m-v & hsa-mir-22 & BCL2 & & & 1 \\
\hline er-alpha-r-v & hsa-mir-369 & ESR1 & & & 1 \\
\hline e-cadherin-r-v & hsa-mir-654 & $\mathrm{CDH} 1$ & & & 1 \\
\hline beta-catenin-r-v & hsa-mir-22 & CTNNB1 & & & 1 \\
\hline $\mathrm{n}$-cadherin-r-v & hsa-mir-511-1 & $\mathrm{CDH} 2$ & & & 1 \\
\hline
\end{tabular}


Citation: Zhu J, Wu S, Yang J (2015) An Integrated Method for Detecting Micro RNA Target Proteins through Reverse-phase Protein Arrays. J Comput Sci Syst Biol 8: 012-033. doi:10.4172/jcsb.1000166

\begin{tabular}{|c|c|c|c|c|c|}
\hline caveolin-1-r-v & hsa-mir-200a & CAV1 & & & 1 \\
\hline chk2-m-c & hsa-let-7b & CHEK2 & & & 1 \\
\hline akt-r-v & hsa-mir-223 & AKT1 & AKT2 & AKT3 & 1 \\
\hline alpha-catenin-m-v & hsa-mir-142 & CTNNA1 & & & 1 \\
\hline igf-1r-beta-r-c & hsa-mir-511-2 & IGF1R & & & 1 \\
\hline bim-r-v & hsa-mir-1295 & BCL2L11 & & & 1 \\
\hline e-cadherin-r-v & hsa-mir-1908 & $\mathrm{CDH} 1$ & & & 1 \\
\hline syk-m-v & hsa-mir-150 & SYK & & & 1 \\
\hline e-cadherin-r-v & hsa-mir-1228 & $\mathrm{CDH} 1$ & & & 1 \\
\hline p70s6k-r-v & hsa-mir-511-2 & RPS6KB1 & & & 1 \\
\hline c-myc-r-c & hsa-mir-486 & MYC & & & 1 \\
\hline b-raf-m-na & hsa-mir-145 & BRAF & & & 1 \\
\hline eef2k-r-v & hsa-mir-1247 & EEF2K & & & 1 \\
\hline igfbp2-r-v & hsa-mir-664 & IGFBP2 & & & 1 \\
\hline p70s6k-r-v & hsa-mir-511-1 & RPS6KB1 & & & 1 \\
\hline er-alpha-r-v & hsa-mir-1910 & ESR1 & & & 1 \\
\hline eef2k-r-v & hsa-mir-487a & EEF2K & & & 1 \\
\hline claudin-7-r-v & hsa-mir-493 & CLDN7 & & & 1 \\
\hline e-cadherin-r-v & hsa-mir-511-2 & $\mathrm{CDH} 1$ & & & 1 \\
\hline yap-r-v & hsa-mir-142 & YAP1 & & & 1 \\
\hline akt-r-v & hsa-mir-511-2 & AKT1 & AKT2 & AKT3 & 1 \\
\hline alpha-catenin-m-v & hsa-mir-223 & CTNNA1 & & & 1 \\
\hline e-cadherin-r-v & hsa-mir-218-1 & $\mathrm{CDH} 1$ & & & 1 \\
\hline akt-r-v & hsa-mir-766 & AKT1 & AKT2 & AKT3 & 1 \\
\hline bim-r-v & hsa-mir-605 & BCL2L11 & & & 1 \\
\hline yap-r-v & hsa-mir-605 & YAP1 & & & 1 \\
\hline erk2-r-na & hsa-mir-223 & MAPK1 & & & 1 \\
\hline beta-catenin-r-v & hsa-mir-146a & CTNNB1 & & & 1 \\
\hline caspase-8-m-c & hsa-mir-511-2 & CASP8 & & & 1 \\
\hline syk-m-v & hsa-mir-541 & SYK & & & 1 \\
\hline er-alpha-r-v & hsa-mir-379 & ESR1 & & & 1 \\
\hline chk2-m-c & hsa-let-7a-3 & CHEK2 & & & 1 \\
\hline chk2-m-c & hsa-let-7a-2 & CHEK2 & & & 1 \\
\hline e-cadherin-r-v & hsa-mir-511-1 & $\mathrm{CDH} 1$ & & & 1 \\
\hline eef2k-r-v & hsa-mir-541 & EEF2K & & & 1 \\
\hline msh6-r-c & hsa-mir-142 & MSH6 & & & 1 \\
\hline chk2-m-c & hsa-let-7a-1 & CHEK2 & & & 1 \\
\hline e-cadherin-r-v & hsa-mir-299 & $\mathrm{CDH} 1$ & & & 1 \\
\hline b-raf-m-na & hsa-mir-605 & BRAF & & & 1 \\
\hline c-met-m-c & hsa-mir-511-2 & MET & & & 1 \\
\hline fak-r-c & hsa-mir-616 & PTK2 & & & 1 \\
\hline pkc-alpha-m-v & hsa-mir-150 & PRKCA & & & 1 \\
\hline bim-r-v & hsa-mir-223 & BCL2L11 & & & 1 \\
\hline syk-m-v & hsa-mir-483 & SYK & & & 1 \\
\hline msh6-r-c & hsa-mir-146a & MSH6 & & & 1 \\
\hline b-raf-m-na & hsa-mir-223 & BRAF & & & 1 \\
\hline igfbp2-r-v & hsa-mir-29b-1 & IGFBP2 & & & 1 \\
\hline b-raf-m-na & hsa-mir-511-2 & BRAF & & & 1 \\
\hline er-alpha-r-v & hsa-mir-218-1 & ESR1 & & & 1 \\
\hline syk-m-v & hsa-mir-766 & SYK & & & 1 \\
\hline b-raf-m-na & hsa-mir-193a & BRAF & & & 1 \\
\hline 53bp1-r-c & hsa-let-7b & TP53BP1 & & & 1 \\
\hline caveolin-1-r-v & hsa-mir-616 & CAV1 & & & 1 \\
\hline rb-m-v & hsa-mir-452 & RB1 & & & 1 \\
\hline er-alpha-r-v & hsa-mir-199a-2 & ESR1 & & & 1 \\
\hline caveolin-1-r-v & hsa-mir-200b & CAV1 & & & 1 \\
\hline c-met-m-c & hsa-mir-511-1 & MET & & & 1 \\
\hline jnk2-r-c & hsa-mir-223 & MAPK9 & & & 1 \\
\hline chk1-r-v & hsa-mir-511-2 & CHEK1 & & & 1 \\
\hline syk-m-v & hsa-mir-511-2 & SYK & & & 1 \\
\hline igf-1r-beta-r-c & hsa-mir-150 & IGF1R & & & \\
\hline
\end{tabular}


Citation: Zhu J, Wu S, Yang J (2015) An Integrated Method for Detecting Micro RNA Target Proteins through Reverse-phase Protein Arrays. J Comput Sci Syst Biol 8: 012-033. doi:10.4172/jcsb.1000166

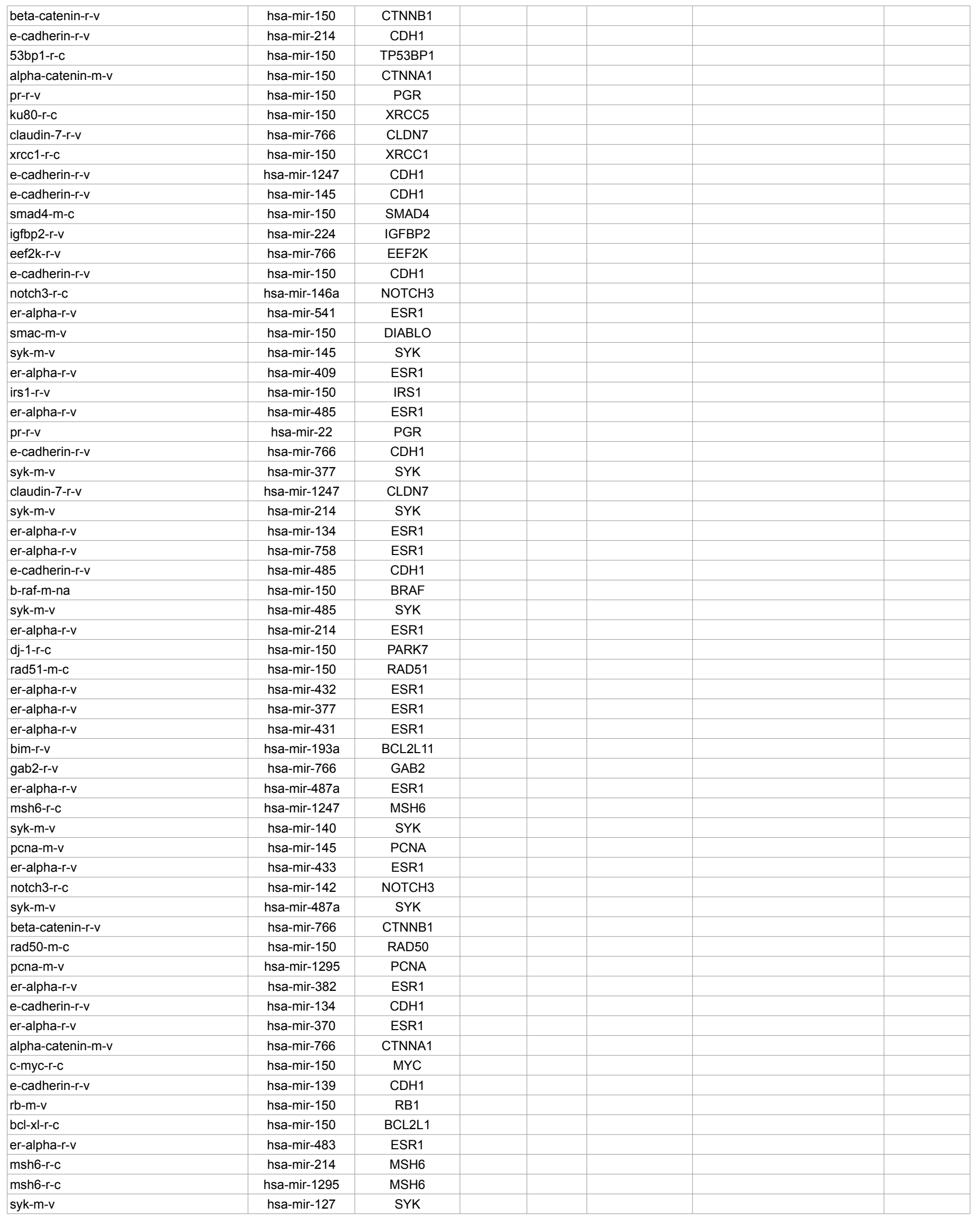


Citation: Zhu J, Wu S, Yang J (2015) An Integrated Method for Detecting Micro RNA Target Proteins through Reverse-phase Protein Arrays. J Comput Sci Syst Biol 8: 012-033. doi:10.4172/jcsb.1000166

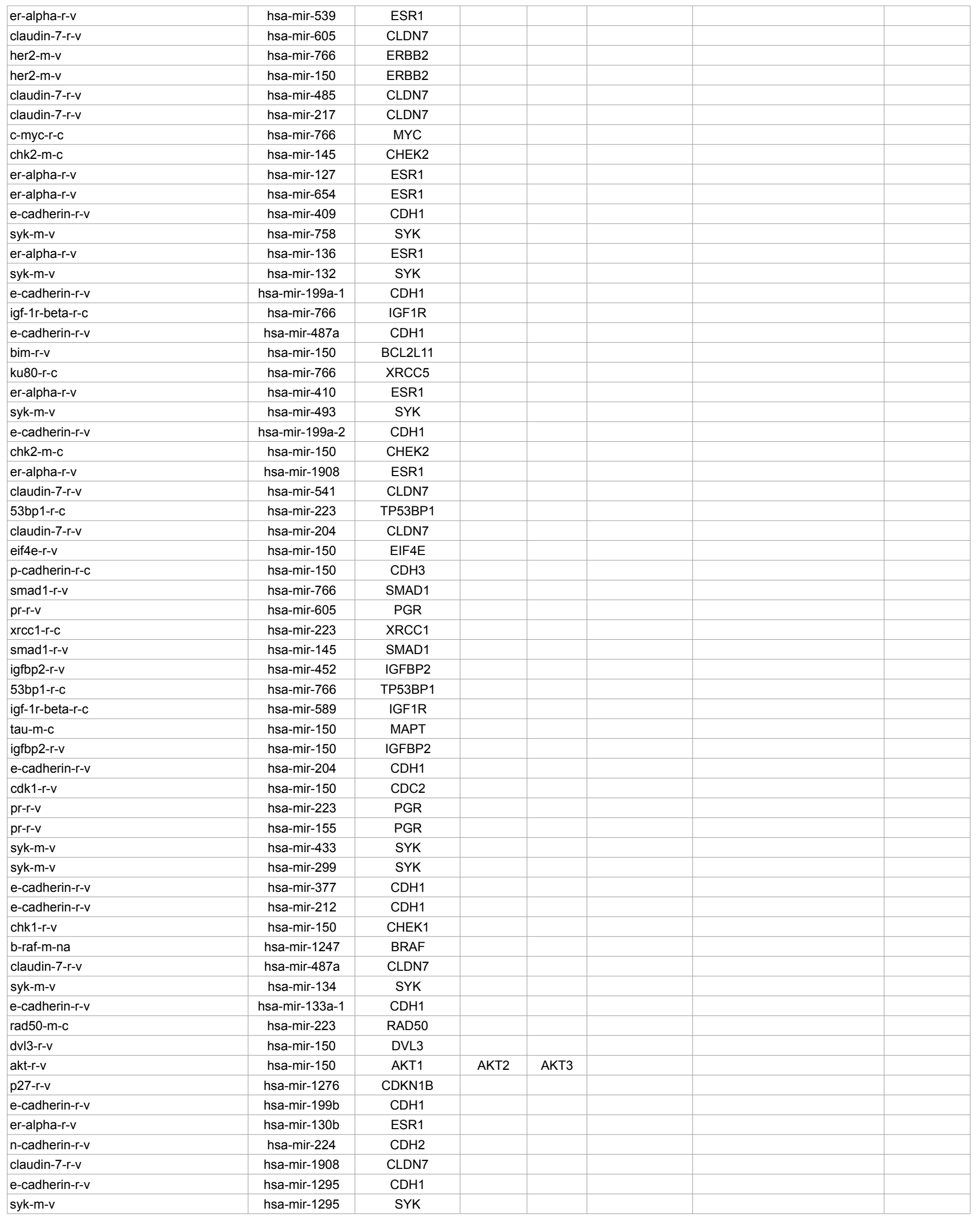


Citation: Zhu J, Wu S, Yang J (2015) An Integrated Method for Detecting Micro RNA Target Proteins through Reverse-phase Protein Arrays. J Comput Sci Syst Biol 8: 012-033. doi:10.4172/jcsb.1000166

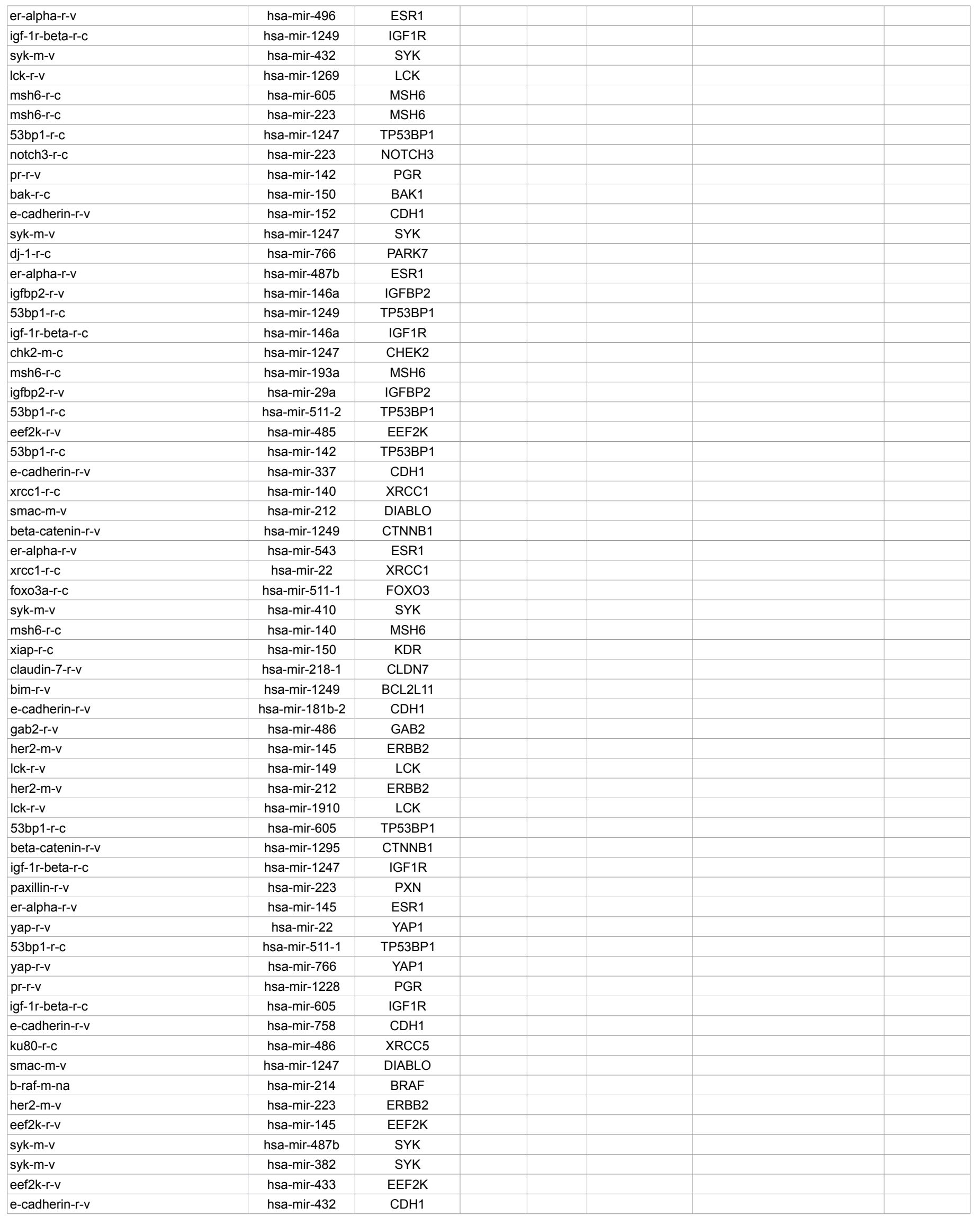


Citation: Zhu J, Wu S, Yang J (2015) An Integrated Method for Detecting Micro RNA Target Proteins through Reverse-phase Protein Arrays. J Comput Sci Syst Biol 8: 012-033. doi:10.4172/jcsb.1000166

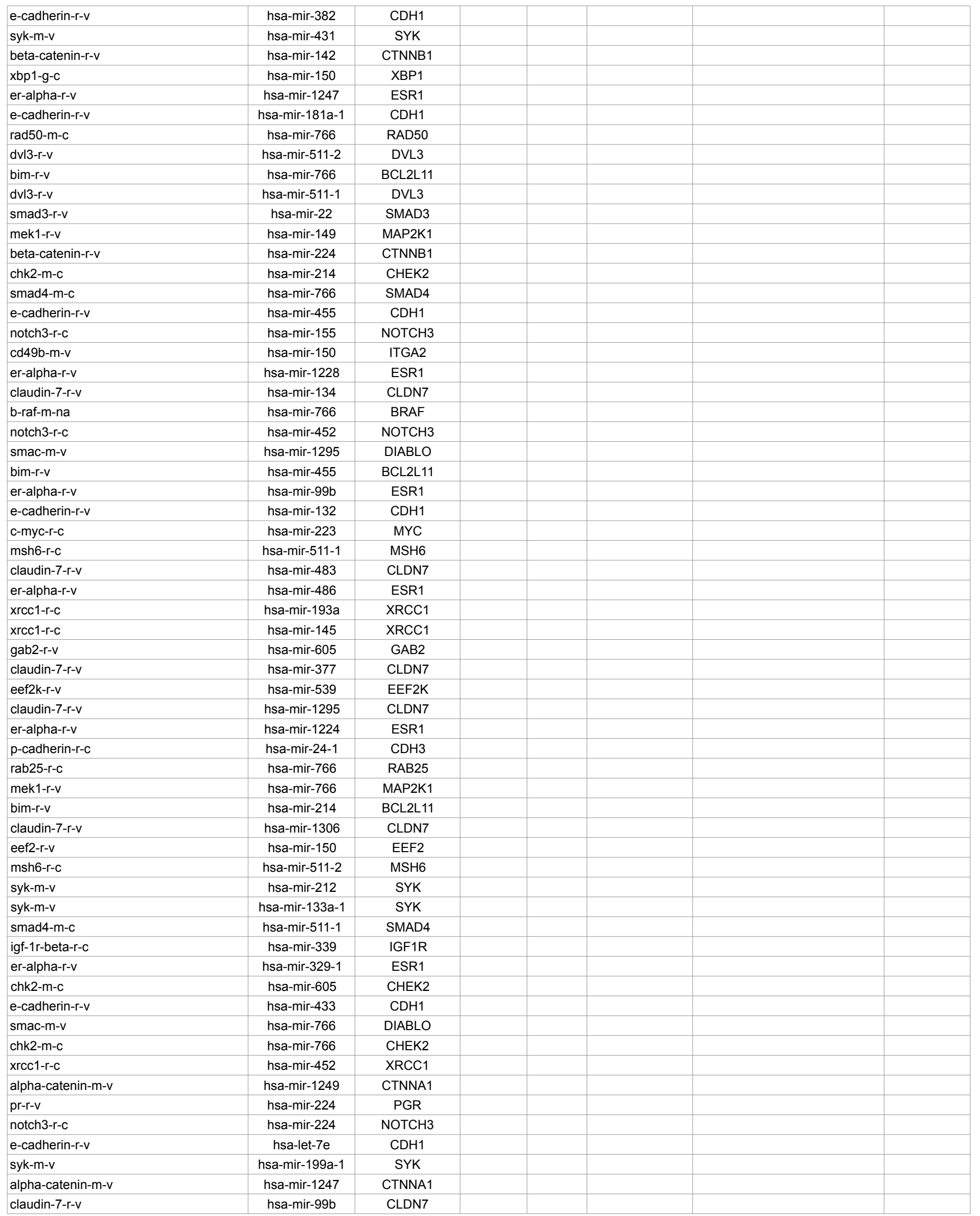


Citation: Zhu J, Wu S, Yang J (2015) An Integrated Method for Detecting Micro RNA Target Proteins through Reverse-phase Protein Arrays. J Comput Sci Syst Biol 8: 012-033. doi:10.4172/jcsb.1000166

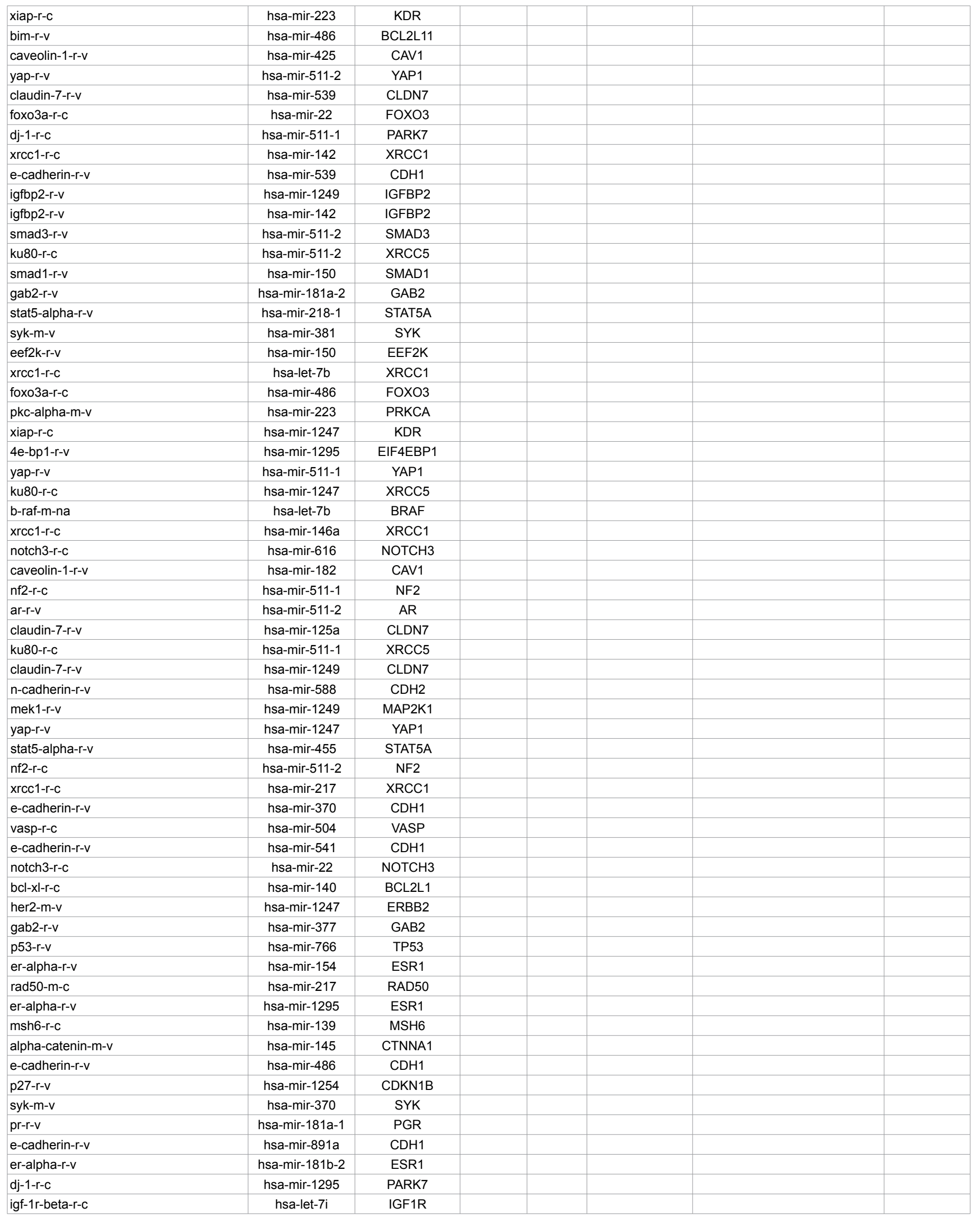


Citation: Zhu J, Wu S, Yang J (2015) An Integrated Method for Detecting Micro RNA Target Proteins through Reverse-phase Protein Arrays. J Comput Sci Syst Biol 8: 012-033. doi:10.4172/jcsb.1000166

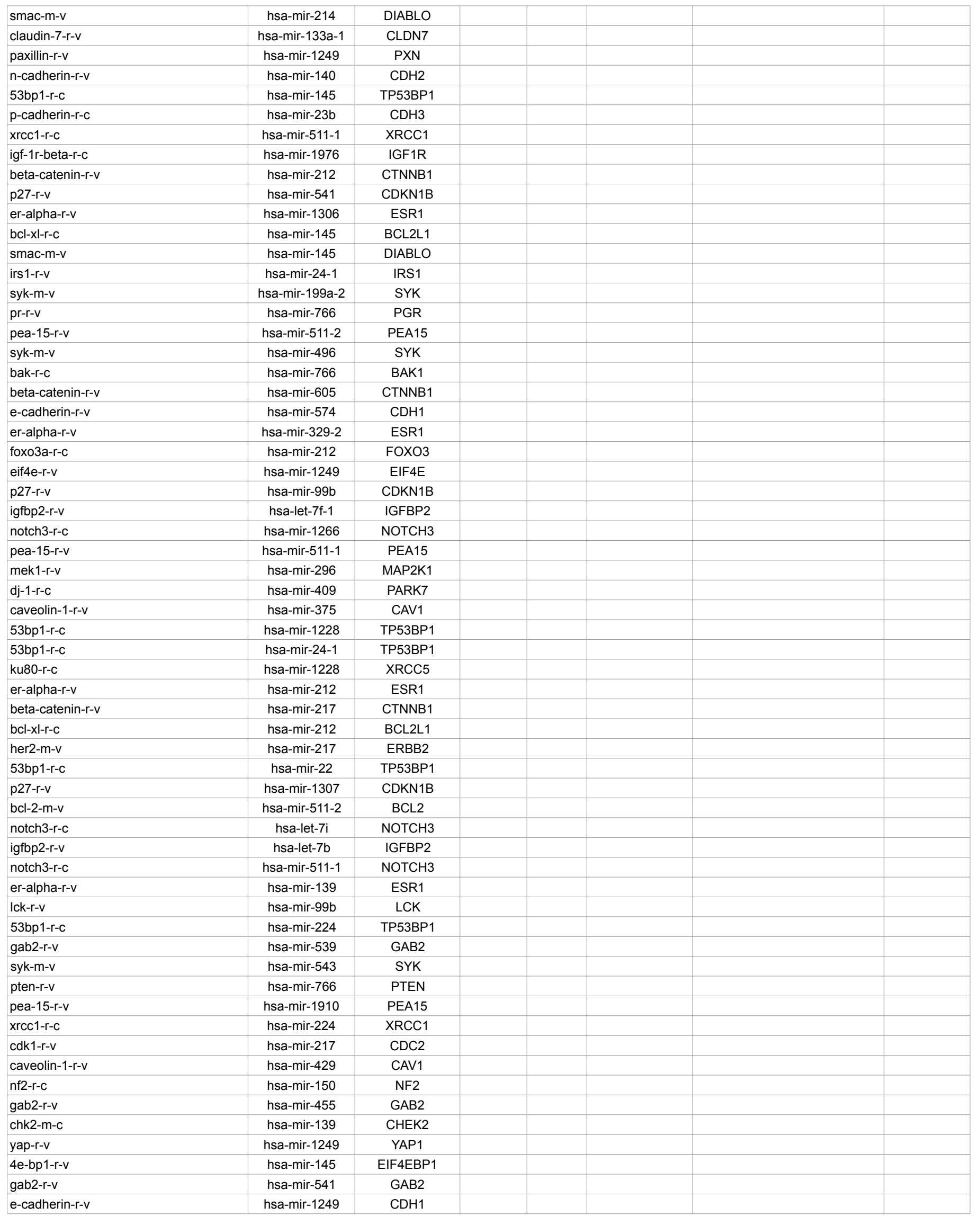


Citation: Zhu J, Wu S, Yang J (2015) An Integrated Method for Detecting Micro RNA Target Proteins through Reverse-phase Protein Arrays. J Comput Sci Syst Biol 8: 012-033. doi:10.4172/jcsb.1000166

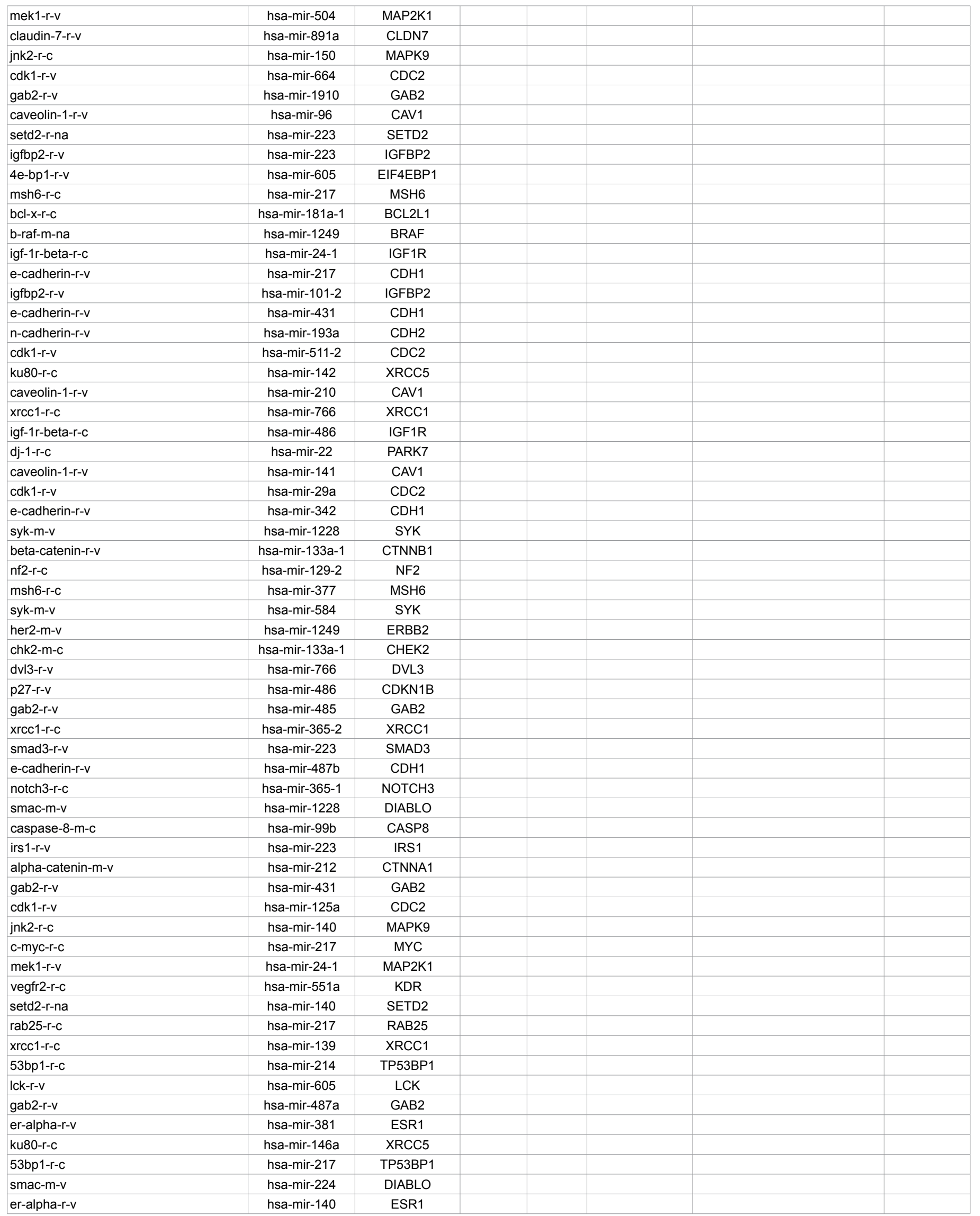


Citation: Zhu J, Wu S, Yang J (2015) An Integrated Method for Detecting Micro RNA Target Proteins through Reverse-phase Protein Arrays. J Comput Sci Syst Biol 8: 012-033. doi:10.4172/jcsb.1000166

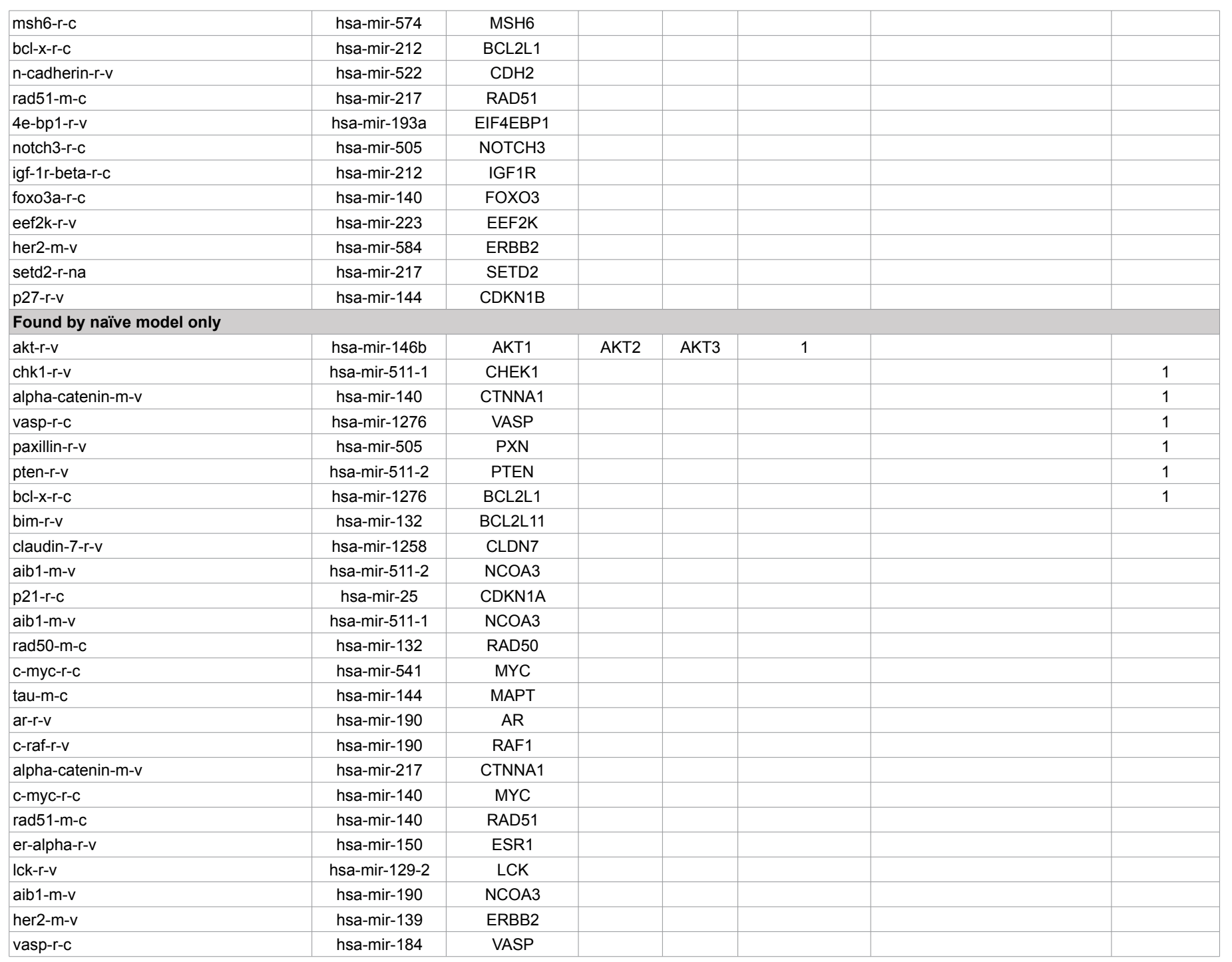

were found only by the integrated model, were confirmed by miRanda database. However, only 6 targets found by the naïve model only were confirmed by miRanda database. MirTarbase, a dataset based on manually surveying pertinent literature [23] was used to further verify our results. 15 suggested targets found by both the integrated and the naïve model were supported by the MirTarBase dataset. 11 of the 15 suggested targets found by both the integrated and the naive model were supported by strong experimental evidences according to the MirTarBase dataset. One suggested target found by the integrated model only were supported by strong experimental evidences according to the MirTarBase dataset. None of the suggested targets found by the naïve model only were supported by strong experimental evidences according to the MirTarBase dataset. This suggests that there could be a number of undiscovered miRNA targets included in the findings of integrated and naïve models. The list of $822 \mathrm{miRNA} /$ protein pairs was included in the appendix (Table A3).

\section{Discussion}

The traditional way to detect direct targets of miRNA using miRNA-mRNA experiment method is limited, due to the fact that miRNAs may regulate their targets post-transcriptionally. In addition, other computational methods, which were based on optimal sequence complementarity of miRNA and mRNA, suffer from large percentage of false positives and of limited practical use. Taking the advantage of recent technique advance in measuring of miRNA expression and protein concentration levels in a high-throughput scale, we proposed to search for potential miRNA targets through a nonlinear hierarchical model. Computationally, this integrated model measures the correlation between miRNA and its targeting protein without making estimation of protein expression levels first as in the naive method. We used both simulation studies and an application to the real data to compare our proposed method and the naïve method. Our simulation results suggested that both integrated and naive methods can well control their type-I errors, however, the integrated method consistently showed higher detection powers than the naive method under different scenarios, particularly when the protein intensity values were located close to the saturation point or the background noise level. In the real data example, our proposed integrated method detected much more potential miRNA targets than the naïve method. Furthermore, the number of potential miRNA targets, which can be confirmed by computational methods or literatures, is larger in the integrated method than that in the naïve method.

A significant association between a miRNA/protein pair can be 
Citation: Zhu J, Wu S, Yang J (2015) An Integrated Method for Detecting Micro RNA Target Proteins through Reverse-phase Protein Arrays. J Comput Sci Syst Biol 8: 012-033. doi:10.4172/jcsb.1000166

either direct or indirect. For example, a miRNA may directly target and degrade a transcription factor (TF), which in turn induces indirect cascading effects of down-regulating the TF's target genes. The association analyses from the simple or our integrated model would reveal both direct and indirect associations. In contrast, the other computer-based algorithms, e.g. miRanda, can only predict direct miRNA targets based on sequence comparison. In the real data analyses (Table 1), the relatively smaller percentage of overlap between our findings and miRanda database suggests that our algorithm may detect more indirect targets. This is sound since our algorithm is more powerful, as demonstrated by our simulation studies, and hence is capable of detecting smaller indirect associations. With the crossreference to miRanda database, those direct miRNA targets of more biological relevance could be filtered out to serve as top candidates for further biological validations. It is worth noting that our algorithm can indeed detect more direct miRNA targets in absolute number. Also, in Table 1, the results were based on a FDR of $10 \%$ for the multiple test adjustment; however, we also checked a FDR at 5\% level and found the conclusion remained the same. That is, the proposed integrated method found more miRNA targets that appear in other existing databases, demonstrating its advantage over the naïve method.

Unknown parameters in our proposed model were estimated within the maximum likelihood framework. Using the asymptotic properties of maximum likelihood estimates, test statistics were straightforward to construct. However, some improvement can be made to further improve the proposed model. For example, we assumed a linear relationship between miRNA and protein to directly compare with the naïve method and to illustrate our model using simple examples, but in reality, the relationship between miRNAs and proteins could follow a nonlinear relationship, such as a dose-response curve. In this case, $\left(z_{i}\right)$ can be replaced by other parametric or nonparametric functions. With some simple modifications, our model can be easily extended to relax these assumptions. Additionally, in this article the random error terms for different dilution steps were set to be independent and identically distributed as proposed in other RPPA analysis papers [10]. However, it is possible that the errors may be highly correlated. In this case, more complicated dependence matrix among serial dilution steps can also be readily incorporated into our model framework.

\section{References}

1. Pritchard CC, Cheng HH, Tewari M (2012) MicroRNA profiling: approaches and considerations. Nat Rev Genet 13: 358-369.

2. Tang G, Reinhart BJ, Bartel DP, Zamore PD (2003) A biochemical framework for RNA silencing in plants. Genes Dev 17: 49-63.

3. Xie Z, Kasschau KD, Carrington JC (2003) Negative feedback regulation of Dicer-Like1 in Arabidopsis by microRNA-guided mRNA degradation. Curr Bio 13: $784-789$

4. Doench JG, Sharp PA (2004) Specificity of microRNA target selection in translational repression. Genes Dev 18: 504-511.

5. Zeng Y, Wagner EJ, Cullen BR (2002) Both natural and designed micro RNAs can inhibit the expression of cognate mRNAs when expressed in human cells. Mol Cell 9: 1327-1333.

6. Betel D, Koppal A, Agius P, Sander C, Leslie C (2010) Comprehensive modeling of microRNA targets predicts functional non-conserved and noncanonical sites. Genome Biol 11: R90.
7. Lewis BP, Shih IH, Jones-Rhoades MW, Bartel DP, Burge CB (2003) Prediction of mammalian microRNA targets. Cell 115: 787-798.

8. Brennecke J, Stark A, Russell RB, Cohen SM (2005) Principles of microRNAtarget recognition. PLoS Biol 3: e85.

9. Mueller C, Liotta LA, Espina V (2010) Reverse phase protein microarrays advance to use in clinical trials. Mol Oncol 4: 461-481.

10. Tabus I, Hategan A, Mircean C, Rissanen J, Shmulevich I, et al. (2006) Nonlinear modeling of protein expressions in protein arrays. IEEE Transactions on Signal Processing 54: 2394-2407.

11. Yang JY, He X (2011) A multistep protein lysate array quantification method and its statistical properties. Biometrics 67: 1197-1205.

12. Hu J, He X, Baggerly KA, Coombes KR, Hennessy BT, et al. (2007) Nonparametric quantification of protein lysate arrays. Bioinformatics 23: 1986-1994.

13. Gelman A, Chew GL, Shnaidman M (2004) Bayesian analysis of serial dilution assays. Biometrics 60: 407-417.

14. Golub G, Pereyra V (2003) Separable nonlinear least squares: the variable projection method and its applications. Inverse problems, 19: R1.

15. Pinheiro JC, Bates DM (1995) Approximations to the log-likelihood function in the nonlinear mixed-effects model. Journal of Computational and Graphical Statistics 4: 12-35

16. Lindstrom MJ, Bates DM (1988) Newton-Raphson and EM algorithms for linear mixed-effects models for repeated-measures data. Journal of the American Statistical Association, 83: 1014-1022.

17. Dennis Jr JE, Schnabel RB (1996) Numerical methods for unconstrained optimization and nonlinear equations. Classics in Applied Mathematics CL16: $x v+375$.

18. Benjamini $Y$, Hochberg $Y$ (1995) Controlling the false discovery rate: a practical and powerful approach to multiple testing. J R Statist Soc 57: 289-300.

19. Enright AJ, John B, Gaul U, Tuschl T, Sander C, et al. (2003) MicroRNA targets in Drosophila. Genome Biol 5: R1.

20. Hofacker IL, Fontana W, Stadler PF, Bonhoeffer LS, Tacker M, et al. (1994) Fast folding and comparison of RNA secondary structures. Monatshefte für Chemie/Chemical Monthly 125: 167-188.

21. Zuker M, Stiegler P (1981) Optimal computer folding of large RNA sequences using thermodynamics and auxiliary information. Nucleic Acids Res 9: 133-148.

22. McCaskill JS (1990) The equilibrium partition function and base pair binding probabilities for RNA secondary structure. Biopolymers 29: 1105-1119.

23. Hsu SD, Tseng YT, Shrestha S, Lin YL, Khaleel A, et al. (2014) miRTarBase update 2014: an information resource for experimentally validated miRNA target interactions. Nucleic Acids Res 42: D78-85. 